\title{
Balance hidrico de un olivar con riego gota a gota. Resultados de cuatro años de experiencias
}

\author{
Félix MORENO, Georges VACHAUD $\left(^{*}\right)$, José MARTIN-ARANDA, Michel VAUCLIN (*) \& Enrique \\ FERNANDEZ \\ con la colaboracion técnica de José Rodriguez, Robert Laty $\left(^{*}\right)$ \\ Instituto de Recursos Naturales y Agrobiologia de Sevilla (CSIC), Apartado 1052, Sevilla, 41080, Espagne \\ (*) Institut de Mécanique de Grenoble (Unité Associée au CNRS), B.P. 68, F 38402 Saint-Martin-d'Hères Cedex
}

RESUMEN

SUMMARY
En el presente trabajo se describe el dispositivo experimental utilizado en un estudio sobre el balance hídrico de un olivar con riego gota a gota y la metodologia puesta a punto a tal efecto, en una parcela de aproximadamente 1 ha. La parcela incluye dos marcos de plantación correspondientes a $5 \times 5 \mathrm{~m}$ y $7 \times 7 \mathrm{~m}$. El cálculo de los aportes hídricos se ha deducido considerando la evaporación media semanal, medida en un tanque clase $\mathrm{A}$, aplicándole el coeficiente 0,4 . Las dosis correspondientes se han obtenido para una superficie equivalente a la sombra del árbol $\left(12 \mathrm{~m}^{2}\right)$.

En relación con el balance hidrico se ha realizado un registro sistemático de parámetros climáticos, medidas del contenido de agua y de presión del agua en el suelo y dosis de riego diarias.

En el trabajo se considera la representatividad espacial de los sitios de medidas, a partir de un análisis geoestadístico de la textura del suelo y de series espaciotemporales de las medidas hídricas.

El cálculo de los términos del balance hidrico se ha efectuado mediante un método de ponderación que tiene en cuenta las zonas afectadas y no afectadas por los aportes localizados de agua durante el período de riego. Asimismo, la estimación del drenaje es objeto de un tratamiento detallado a partir de las caracteristicas hidrodinámicas del suelo.

A título de ejemplo, se exponen los resultados obtenidos, aplicando el método a punto, en el caso del marco de plantación $7 \times 7 \mathrm{~m}$ y durante dos de los años del período experimental, caracterizados por una considerable diferencia en su pluviometría.

En la última parte de este trabajo se muestran los resultados obtenidos, en términos de balance hídrico, correspondientes a cuatro años de experiencias que pretenden caracterizar la influencia del riego en cada uno de los marcos de plantación utilizados.

Los resultados del balance hídrico ponen de manifiesto la eficacia del riego en ambos marcos de plantación, en relación a la climatologia del año.

Si el aporte hídrico se calcula en relación a la superficie plantada en lugar de árbol individual, la dosis utilizada (0.4 EV del tanque) se convierte en una dosis de $0,2 \mathrm{EV}$ del tanque para un marco de plantación de $5 \times 5 \mathrm{~m}, \mathrm{y}$ de $0,1 \mathrm{EV}$ del tanque para un marco de $7 \times 7 \mathrm{~m}$.

Durante el periodo experimental, se observan diferencias entre las cantidades de agua evapotranspirada por cada uno de los marcos de plantación. Sin embargo, dichas cantidades son muy similares, de un año a otro, dentro de un mismo marco.

El estudio realizado permite establecer los sitios de medida representativos del comportamiento medio de la parcela. Por otra parte, el método de ponderación utilizado es válido para determinar la evapotranspiración real y caracterizar el flujo de drenaje. Por otra parte, la estimación climatológica de la evapotranspiración potencial, unida a las medidas de balance hídrico del suelo, permiten estimar la magnitud de la escorrentía durante lluvias de cierta intensidad.

Palabras clave : Balance hidrico, cargo hidraulica, drenaje, variabilidad espacial, evapotranspiracion, perfil hidrico, textura.

In the present work the experimental design used in the study on water balance in an olive orchard under drip irrigation is described as well as the methodology developed to that end. The experimental plot, 1 ha approx., included two different plant-spacings : $5 \times 5$ and $7 \times 7 \mathrm{~m}$. The amount of water to be applied was calculated considering the total weekly evaporation of a class A pan (EV pan), adjusted by a reduction coefficient. Only the area covered by the tree shadow was taken into account, that is $12 \mathrm{~m}^{2}$. The irrigation dose taken here into account corresponded to the equivalent of $0.4 \mathrm{EV}$. Systematic records of climatic parameters, soil water content, soil water tension and daily irrigation doses were carried out throughout the irrigation period. The spatial representativity of measurement sites was considered in terms of a previous geostatistical analysis of soil texture and of space-time series of water measurements. Water balance parameters were calculated by a model taking into consideration both the areas affected and non-affected by the irrigation water. This model included a ponderation method for changes of water storage and drainage in the mentioned areas. Results of water balance given in this work correspond to four years of experiments and were intended to characterize the influence of irrigation on each of the two plant spacings used. Water balance results showed the efficiency of irrigation on both plots, according to climatology. Irrigation efficiency was strongly affected by soil water content at the beginning of the irrigation 
period. When the amount of water applied was referred to the total planted area instead of the individual tree, the dose used (0.4 EV pan) was equivalent to $0.2 \mathrm{EV}$ in treatment $5 \times 5 \mathrm{~m}$ and $0.1 \mathrm{EV}$ pan in treatment $7 \times 7 \mathrm{~m}$. Differences of evapotranspiration values were observed between the two plant-spacings, but little difference was found for different years within the same plot. The study carried out allowed us to establish sites that were representative for the mean behaviour of the plot. The ponderation method used was valid for determining actual evapotranspiration and characterizing drainage flux. The estimation of potential evapotranspiration, together with measurements of soil water balance parameters, permitted estimation of water run-off during periods of intense rainfall.

Additionnal key words : Water balance, hydraulic head, drainage, spatial variability, evapotranspiration, water content profile, texture.

\section{RÉSUMÉ}

\section{Bilan hydrique d'une oliveraie irriguée au goutte à goutte. Résultats de quatre années d'expéri- mentation.}

Cette étude a été effectuée dans le cadre d'une Action Intégrée de Recherche Franco-Espagnole, avec pour objectif la mise au point d'une méthode permettant d'estimer la consommation d'eau d'une oliveraie irriguée au goutte à goutte afin de préconiser une minimisation des apports d'eau pour une qualité donnée du produit (olive de table). Pour ce faire, on s'est placé dans le cas d'une exploitation traditionnelle d'Andalousie, en considérant les 2 densités de plantation les plus généralement utilisées (un arbre tous les 25 ou $49 \mathrm{~m}^{2}$, soit une interdistance de 5 ou $7 \mathrm{~m}$ entre arbres). Le calcul des apports a été déterminé par l'évaporation moyenne hebdomadaire d'un bac classe A (EV Bac) affectée éventuellement d'un facteur de minoration et ramenée à une lame d'eau équivalente rapportée à la surface de "l'onbre d'un arbre », soit environ $12 \mathrm{~m}^{2}$. La dose d'irrigation, calculée au départ à $0,8 \mathrm{EV}$ Bac, a été rapidement réduite à $0,4 \mathrm{EV}$ Bac compte tenu de la mise en évidence de percolations en profondeur très importantes. On a mis enfin à profit les possibilités offertes par le systeme d'irrigation pour tester également l'effet de la fertilisation en utilisant, à mème apport d'eau, différentes doses de solution nutritive. L'expérimentation recouvre 4 années d'observations. Cet article concerne la description de la méthodologie utilisée pour rechercher des sites de mesures représentatifs à l'échelle de l'expérimentation (environ 1 ha), tenant compte de la variabilité spatiale des transferts dans le sol, et calculer le bilan hydrique en tenant compte de la présence d'un "bulbe " autour des arbres; puis un exemple d'application portant sur l'estimation du bilan hydrique durant 2 années consécutives, mais très contrastées du point de vue hydrologique; et enfin l'analyse des résultats pluriannuels.

Plusieurs types de mesures, pour la plupart implantés lors d'une expérimentation précédente sur le même verger, sont disponibles; essentiellement 3 séries d'informations :

- mesures bioclimatiques, sur une base journalière, à partir d'une station agrométéorologique classique, accompagnees de mesures journalières des apports d'irrigation ;

- mesures hydriques dans le sol, portant sur les teneurs en eau par humidimètre neutronique, et sur les pressions d'eau, par tensiomètres, sur des sites installés autour de certains arbres (jusqu'à 3 ou 4 sites par arbres, implantés à $0,5,1,5,2,5$ et $3,5 \mathrm{~m}$ du tronc pour des interdistances de 5 et $7 \mathrm{~m}$ ). Ces mesures sont effectuées toutes les semaines en période d'irrigation (en général avril-mai à octobre), tous les mois hors irrigation autour des 15 arbres ;

- mesures physiologiques sur les arbres donnant arbre par arbre la croissance et le nombre de rameaux, des paramètres de structure foliaire, le nombre et le poids des olives.

On s'est d'abord attaché à rechercher pour chaque densité de plantation s'il était possible de sélectionner un arbre ayant fait l'objet d'une instrumentation dans le sol que l'on puisse considérer comme représentatif de l'expérimentation. Tirant profit de résultats analogues obtenus précédemment (VACHAUD et al., 1985), on a d'abord effectué une analyse géostatistique portant sur la texture du sol qui a conduit à retenir des emplacements de mesures pouvant avoir un comportement qualifié de «moyen». Une analyse, a posteriori, des séries de mesures spatio-temporelles d'humidité a permis de valider la représentativité des sites ainsi choisis au niveau de l'estimation des variations de stock d'eau du sol.

Sur chacun des 2 emplacements ainsi retenus (un pour chaque densité de plantations), on a effectué une caractérisation hydrodynamique afin d'obtenir la relation conductivité-teneur en eau $(\mathrm{K}(\theta))$ permettant d'effectuer le calcul des percolations par application de la loi de Darcy. On s'est ensuite attaché à développer une méthode d'estimation du bilan hydrique à l'échelle d'une maille (25 ou $49 \mathrm{~m}^{2}$ autour d'un arbre) en s'appuyant sur le fait qu'il existe toujours, en période d'irrigation, 2 zones très distinctes dans cette maille : l'une, de dimension à peu près constante $(4 \mathrm{~m}$ de diamètre environ) affectée par l'irrigation, et dans laquelle se trouvent toujours 2 tubes d'accès pour humidimètre neutronique ; l'une qualifiée de zone « sèche ». En faisant en outre l'hypothèse que dans la zone affectée par l'irrigation (le «bulbe ») l'on est dans un système axisymétrique, on propose une méthode de pondération des mesures neutroniques (stock d'eau) fonction des surfaces affectées à chacun des tubes d'accès implantés autour de l'arbre. Ainsi, on suppose que le $1^{\text {er }}$ tube $(a ̀ ~ 0,5 \mathrm{~m})$ caractérise un cylindre de $1 \mathrm{~m}$ de diamètre centré autour de l'arbre; le second $(1,5 \mathrm{~m})$ un anneau de diamètre intérieur et extérieur 1 et $2 \mathrm{~m}$. Ces 2 tubes, selon la formule de pondération donnée (équation 1), qui ne prend en compte que les surfaces correspondantes, permettent d'obtenir un comportement équivalent de la zone affectée par l'irrigation (indice i). Dans la zone dite sèche (non irriguée, indice $n, i$ ), les mesures obtenues sur les 2 tubes (cas d'une maille $7 \times 7 \mathrm{~m}$ ) sont en général très voisines, ce qui permet une simple formule de moyenne (eq. 2). Enfin, les termes de bilan, ramenés à l'échelle d'une maille, prennent en compte le rapport des surfaces des zones irriguée et sèche et s'expriment par les formules (3) et (4) dans le cas d'une maille de $5 \times 5 \mathrm{~m}$ et de $7 \times 7 \mathrm{~m}$ réciproquement.

Dans ces calculs, le drainage (à la cote $2,4 \mathrm{~m}$ ) est obtenu par application de la loi de Darcy ; le ruissellement à partir d'un algorithme basé sur une comparaison entre ETR et ETP Penman.

On notera que les pondérations s'appliquent aux valeurs de variations de stock, et de lame drainée obtenues sur chaque tube d'accès.

On donne à titre d'application les résultats obtenus dans le cas de la maille $7 \times 7 \mathrm{~m}$ en 1983 et 1984 , avec une pluviométrie de 258 , puis de $543 \mathrm{~mm}$. On relèvera, parmi les points les plus importants, que l'évapotranspiration réelle est à peu près la même (environ $330 \mathrm{~mm}$ ). Au niveau du bilan hydrique les différences essentielles concernent l'occurrence de très forts ruissellements en 1984, que la méthock permet d'estimer à environ $85 \mathrm{~mm}$, et surtout un doublement des pertes en drainage qui, en 1984, correspondent i peu près aux apports en irrigation. Les mesures tensiométriques montrent ainsi que pendant toute la période dirrigation en 1984 le sol est saturé, près du tronc, jusqu'à une profondeur de $0,75 \mathrm{~m}$. 
Dans la dernière partie, on s'attache à définir, au terme de l'expérimentation de 4 ans, l'efficacité de la méthode d'irrigation pour chacune des 2 densités de plantation étudiées.

Les fortes variations interannuelles de pluviométrie permettent de mettre clairement en évidence l'importance de l'humidité du sol sur les pertes en drainage. Les bilans effectués chaque année montrent que la méthode de calcul des doses traditionnelles, qui ne tient compte que de facteurs climatiques et non de l'état hydrique du sol, conduit après un hiver humide, à des pertes en drainage voisines des apports d'irrigation.

On notera en outre que ces résultats ont été obtenus en utilisant un coefficient de réduction de dose $(0,4 \mathrm{EV}$ Bac), alors qu'en général au niveau des exploitations andalouses, on applique directement une dose par arbre égale à l'Evaporation Bac. Il apparaît donc clairement que des économies très importantes d'eau peuvent être réalisées. Si l'on se replace au niveau d'un contexte plus hydrologique, revenant à calculer l'apport d'eau non pas par arbre, mais par surface plantée, on notera que la dose utilisée $(0,4 \mathrm{EV}$ Bac par arbre) revient en fait à une dose de $0,2 \mathrm{EV}$ Bac pour une maille de $5 \times 5 \mathrm{~m}$, et de $0,1 \mathrm{EV}$ Bac pour une maille de $7 \times 7 \mathrm{~m}$. Il s'agit donc d'apports faibles mais qui pourraient encore être substantiellement optimisés si l'on prenait en compte un critère d'humidité du sol.

L'effet «densité de plantation» est relativement secondaire au niveau de l'évapotranspiration, qui est toutefois légèrement supérieure sur le traitement $5 \times 5 \mathrm{~m}$. On notera en outre que la variabilité interannuelle de l'ETR est également faible malgré les fortes différences de pluviométrie. C'est essentiellement le drainage qui varie.

On a enfin tenté de déterminer une relation entre consommation en eau et production. L'analyse est complexe pendant une aussi courte période marquée par la persistance de l'alternance de production et l'effet de taille sévère. Il n'est pas non plus évident que les arbres aient eu le temps de réagir aux variations d'irrigation imposées en début d'essai.

L'essai continue pour obtenir des résultats concluants à ce niveau. Il paraît néanmoins évident que le mode d'irrigation au goutte à goutte, actuellement utilisé dans les grandes exploitations andalouses, est mal adapté à cette culture et peut être profondément amélioré par la connaissance des transferts dans le sol.

Mots clés additionnels : Bilan hydrique, charge hydraulique, drainage, variabilité spatiale, évapotranspiration, profil hydrique, texture.

\section{INTRODUCCION}

En la cuenca mediterránea, el agua disponible para las plantas es el factor limitante de mayor importancia para obtener una produccion regular y de calidad. El desarrollo de investigaciones basadas en la determinación de métodos de riego correspondientes a una minimización de los aportes de agua para una calidad dada del producto es, pues, prioritario. Este aspecto es de particular interés en el cultivo en riego del olivar de mesa, que ha experimentado un fuerte incremento, donde el tamaño del fruto puede estar profundamente condicionado por el estrés hídrico.

Se sabe que la alimentación hídrica de las plantas tiene influencia sobre la fructificación, así como que la respuesta del árbol depende de manera especial del clima, del suelo y de la variedad. Hay que hacer notar por otra parte, que, en el caso del olivo, existen datos contradictorios en la bibliografia acerca del efecto del riego sobre la alternancia en la producción (SERRANO y Abela, 1982, 1983, 1984; Serrano y Delteil, 1981 ; LE BouRdELLES, 1979).

La introducción del riego gota a gota en plantaciones de olivar en Andalucía ha alcanzado una gran difusión, sin que se hayan realizado estudios sistemáticos de las necesidades hídricas de este cultivo. El Centro de Edafología y Biología Aplicada del Cuarto de Sevilla (CSIC) creyó conveniente realizar un estudio en este sentido y para ello en 1979 se pusieron en marcha experiencias con objeto de caracterizar el suelo y el medio en una primera etapa. El interés de los resultados obtenidos, publicados por NuÑEZ y col., 1981 ; MARTIN ARANDA y col., 1982, condujeron a ampliar los trabajos emprendidos a fin de determinar los balances hídricos.

El estudio que se llevó a cabo, cuyos resultados se describen a continuación, se realizó en el marco de una Acción Integrada hispano-francesa, entre el citado Centro español (actualmente Instituto de Recursos
Naturales y Agrobiología de Sevilla) y el Institut de Mécanique de Grenoble (Laboratorio asociado del CNRS), entre 1981 y 1986.

Las primeras conclusiones permiten considerar que las dosis de riego normalmente utilizadas en nuestra región, en plantaciones de olivar con riego gota a gota, pueden ser excesivas e inducir a pérdidas importantes tanto de agua como de elementos minerales.

\section{MATERIAL Y METODOS}

\section{A. Parcela experimental-suelo y clima}

La experiencia que se presenta en este estudio comenzó en 1979 y ha sido realizada en la finca experimental Aljarafe, perteneciente al CSIC, situada a $20 \mathrm{~km}$ al suroeste de Sevilla, en la que se ha dispuesto de una parcela cuya superficie es de 1 ha, aproximadamente, de olivar de mesa (Olea europea L., variedad manzanillo), de 16 años de edad, con dos marcos de plantación : $5 \times 5 \mathrm{~m}$ y $7 \times 7 \mathrm{~m}$ y con riego gota a gota. Se ha dispuesto, asimismo, de una parcela testigo, sin riego, con marco de plantación de $7 \times 7 \mathrm{~m}$.

La parcela está situada en una zona ondulada, del Mioceno del Aljarafe, con una pendiente media del $3 \%$, siendo el material original arenisca caliza. El suelo está clasificado como un Xerochrept (Soil Taxonomy) y posee una textura media de $22,6 \%$ de arcilla, $13,4 \%$ de limo, $36,5 \%$ de arena fina y $27,5 \%$ de arena gruesa.

La zona posee un clima típicamente mediterráneo, con lluvias de invierno y primavera (media anual $500 \mathrm{~mm}$ ) y veranos muy calurosos y secos. A pie de la parcela se cuenta con una estación meteorológica estandard. Las lecturas se realizan diariamente. La evapotranspiración potencial se ha calculado por períodos 

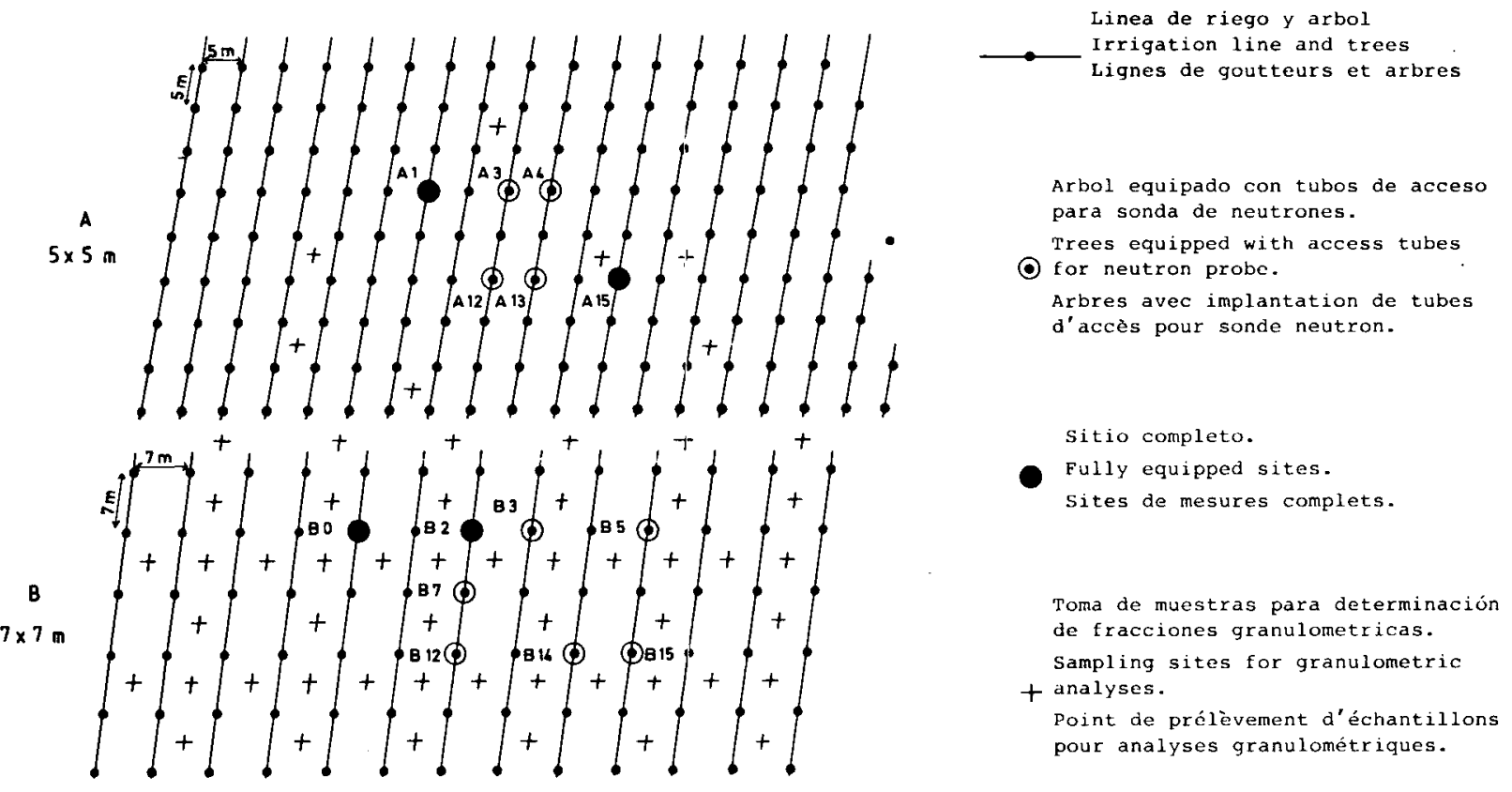

Figure 1

Esquema de la parcela experimental.

Experimental lay-out.

Schéma de l'expérimentation.

decenales a partir de la fórmula de Penman. Los datos detallados correspondientes al período experimental se encuentran en el trabajo de MORENO y col. (1987).

\section{B. Diseño y metodo de riego}

La parcela está equipada con un sistema de riego localizado gota a gota, con líneas dispuestas segúr la dirección de la pendiente más importante del terreno. Sobre el marco de plantación $5 \times 5 \mathrm{~m}$ (subparcela A) se han implantado 18 líneas de $40 \mathrm{~m}$ de longitud cada una, y en el marco $7 \times 7 \mathrm{~m}$ (subparcela B), 13 líneas de $32 \mathrm{~m}$ (fig. 1). El conjunto está conectado a una bomba, con sectorización en 4 zonas y regulación automática del tiempo de riego y del aporte de fertilizante (bomba de inyección de fertilizante) independiente para cada una de las zonas. El riego se efectúa sistemáticamente durante cada noche en la forma que se describe más adelante. Cada árbol está provisto de cuatro goteros (dos a cada lado del árbol, según la línea de goteros), de tipo key-clip, con una distancia entre goteros de $1 \mathrm{~m}$.

Las dosis de riego han sido calculadas según un método ya habitual consistente en utilizar el equivalente de la evaporación medida en el tanque en la semana precedente, afectado de un coeficiente de reducción y aplicando la cantidad de agua correspondiente sobre una superficie que es igual a la sombra del árbol, y que en nuestro caso representa, por término medio, un círculo de $4 \mathrm{~m}$ de diámetro $\left(12,5 \mathrm{~m}^{2}\right)$, de forma que el aporte diario de agua correspondiente era programado cada semana de acuerdo con las características del gotero empleado, que proporciona un caudal de $4 \pm 0,1 \mathrm{l} / \mathrm{h}$. La validez de este método será discutida en otro apartado. La solución nutritiva era incorporada al agua de riego mediante una bomba de inyección situada a continuación de la bomba de alimentación.
Dentro del presente estudio cabe diferenciar dos fases sucesivas :

- De mayo de 1981 hasta octubre de 1983, en que el riego ha sido efectuado con las dosis correspondientes a los niveles 0,4 y 0,8 de la evaporación del tanque y con dos tasas de fertilización $F_{1}$ y $2 F_{1}$, donde $F_{1}$ corresponde a una concentración de $0,6 \mathrm{~kg}$ de nitrógeno, $0,05 \mathrm{~kg}$ de fósforo y $0,35 \mathrm{~kg}$ de potasio por árbol durante el período de riego.

- Desde octubre de 1983, en que teniendo en cuenta la importancia de las pérdidas aparentes por drenaje, y la ausencia de repetición de medidas en el suelo sobre el tratamiento 0,8 EV (tanque), un solo nivel de aporte de agua $(0,4 \mathrm{EV}$ tanque) ha sido empleado en toda la parcela, pero con diferentes tasas de fertilización $F_{1}$, $2 \mathrm{~F}_{1}, 3 \mathrm{~F}_{1}$ y $\mathrm{F}_{0}$, donde $\mathrm{F}_{0}$ corresponde a una fertilización cero.

En la presente exposición sólo se condiserarán los resultados obtenidos a partir del tratamiento $0,4 \mathrm{EV}$.

\section{Medidas consideradas y frecuencia}

Para el período de estudio indicado se ha dispuesto de diferentes series de medidas, las cuales aportan la siguiente información :

- Medidas bioclimáticas diarias, en una estación agrometeorológica estandard, y medidas diarias de los aportes hídricos mediante riego.

- Medidas fisiológicas en la planta, considerando para cada árbol el crecimiento y el número de ramos, parámetros de estructura foliar y el número y peso de los frutos.

- Medidas hídricas en el suelo, efectuadas con una sonda de neutrones, y medida de la presión del agua en 

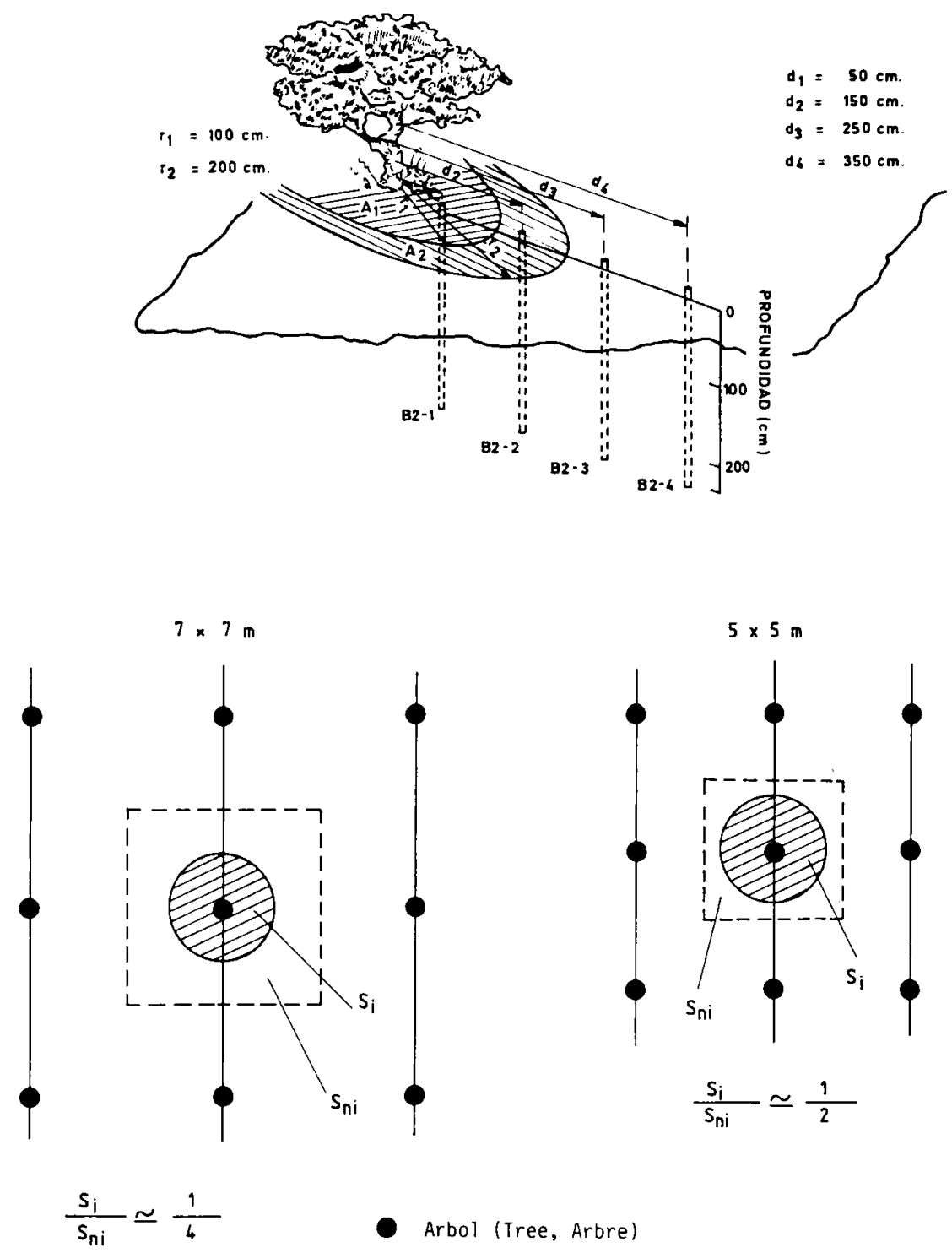

Figure 2

Disposicion de los tubos y definicion de las superficies regadas y no regadas.

Location of tubes and definition of irrigated and non-irrigated areas.

Disposition des tubes et caractérisation des surfaces de mailles irriguées et non irriguées.

el suelo mediante tensiometría, en diferentes puntos de medida situados alrededor de ciertos árboles ( 3 ó 4 puntos por árbol, a distancias de 0,$5 ; 1,5 ; 2,5$ y $3,5 \mathrm{~m}$ del tronco, para distancias entre árboles de 5 y $7 \mathrm{~m}$ ) (fig. 2). Estas medidas han sido realizadas semanalmente durante el período de riego (en general de abril-mayo a octubre) y mensualmente en el resto del año, alrededor de 15 árboles.

En cada una de las subparcelas existe un número limitado de sitios completamente equipados (puntos negros de la fig. 1) con tubos que permiten la medida del perfil hídrico hasta 2,4 m de profundidad : Al y A15, en el marco de plantación $5 \times 5 \mathrm{~m}$; y BO, B2 y B15, en el marco de plantación $7 \times 7 \mathrm{~m}$. Los sitios restantes poseen tubos de acceso cuya profundidad útil es de $1,5 \mathrm{~m}$. Los detalles de instalación pueden verse en un trabajo anterior (MORENo y col., 1987).

Para el manejo de magnitudes observadas, teniendo en cuenta el elevado número de medidas de contenido de agua del suelo obtenidos (alrededor de 30000 ), se ha aplicado un programa de adquisición y tratamiento de datos (AIDHYS) desarrollado a tal fin (LATY y VACHAUD, 1987).

\section{Representatividad espacial de los sitios de medida}

La elección de los sitios de medida se hizo considerando los árboles que eran representativos del conjunto de las dos subparcelas. A continucación se efectó un doble análisis para validar la elección.

Por una parte, un análisis geoestadístico de la textura, teniendo en cuenta las fuertes relaciones entre ésta y las características hidrodinámicas (VAN GENUCHTEN, 1980 ; Bloemen, 1980 ; Haverkamp y Parlange, 1986) y por otra parte, un estudio de series de medidas espacio-temporales en cada tratamiento.

La caracterización textural se ha llevado a cabo a partir del análisis de 57 perfiles de suelo, de los cuales 48 


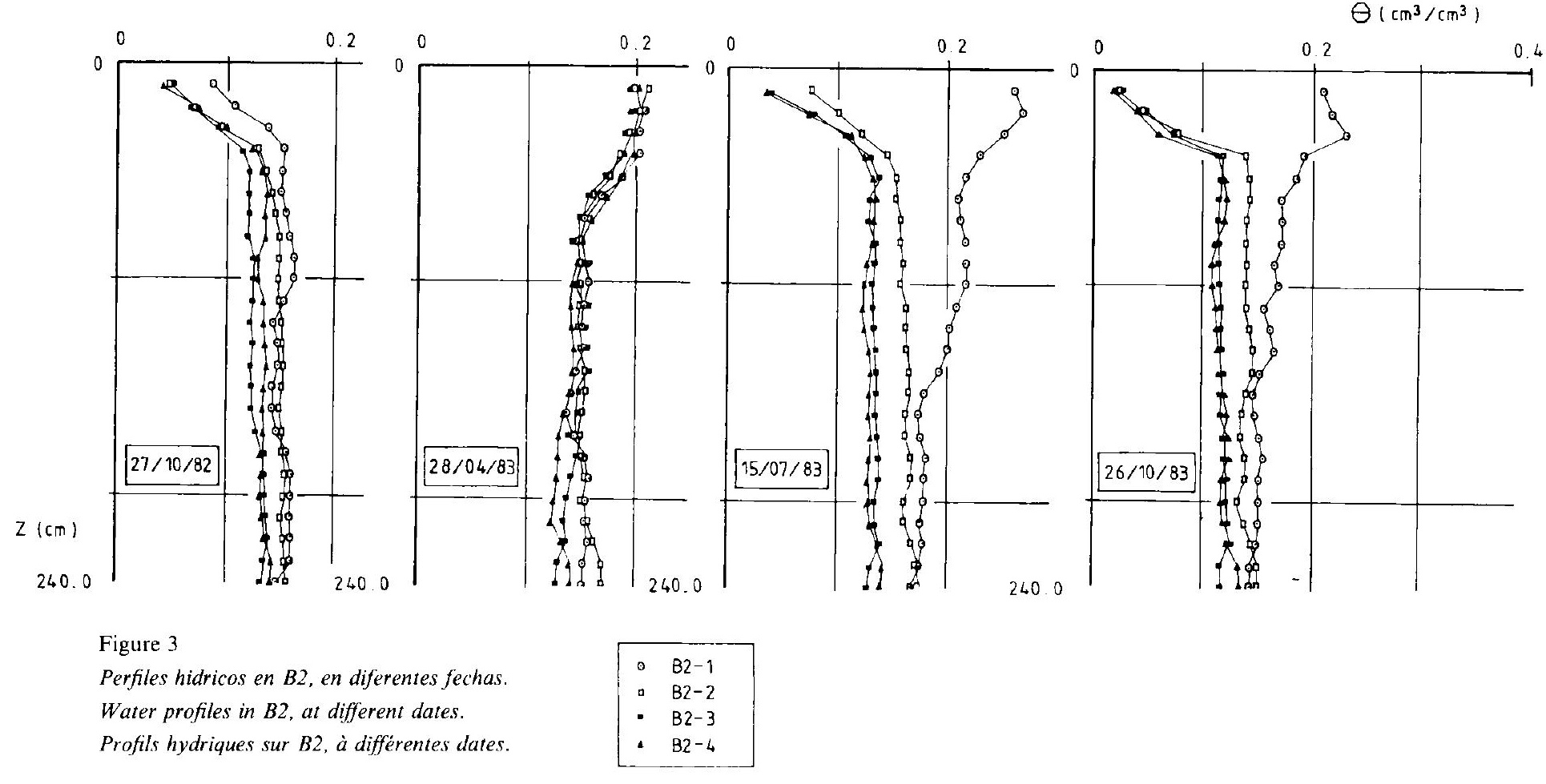

corresponden a una malla regular (fig. 1). En cada uno de estos perfiles se tomaron muestras, con barrena, a intervalos de $0,25 \mathrm{~m}$ y hasta una profundidad de $1,25 \mathrm{~m}$, determinándose en cada una la composición granulométrica (fracciones $>200 \mu \mathrm{m}, 200-20 \mu \mathrm{m}, 20-2 \mu \mathrm{m}$ y $<2 \mu \mathrm{m}$ ). Los resultados, detallados en el trabajo de MORENo y col. (1987), conducen esencialmente a las conclusiones siguientes:

- La variabilidad vertical es, prácticamente, despreciable frente a la variabilidad horizontal. Por tanto, para cada punto, se pueden utilizar las características granulométricas medias del perfil.

- Las fracciones de limo y arena gruesa pueden caracterizarse muy bien por el parámetro $\mathrm{d}_{50}$ (diámetro medio, correspondiente al $50 \%$ en peso de las partículas), con los coeficientes de correlación cruzados de 0,84 y 0,87 .

La fuerte variabilidad del parámetro $\mathrm{d}_{50}$, que ciertos autores (BLOEMEN, 1980) preconizan para predecir las curvas $\mathrm{k}(\theta)$, aconseja ser muy cuidadosos sobre la extrapolación, a nivel de la parcela, de medidas puntuales de humedad obtenidas con una sonda de neutrones. La carta de isovalores obtenida por «krigeage" (Moreno y col., 1987) muestra, no obstante, que los sitios A15 y B2, escogidos en principio como árboles representativos de los respectivos marcos de plantación, están situados en una zona que caracteriza bien la probabilidad de que se dé el valor medio de $\mathrm{d}_{50}$ sobre la parcela, lo que apoya la hipótesis de que estos sitios representan un comportamiento medio.

Para apoyar esta opinión, hemos efectuado otro estudio sistemático que permite situar, en cada instarte, la medida hídrica en estos dos sitios, en relación a todas las medidas obtenidas al mismo tiempo sobre el resto de los sitios de la parcela. Este análisis, que ha dado lugar a otra publicación (VACHAUD y col., 1985a), muestra que, para el árbol B2, durante el período 1981 a 1985 los contenidos totales de agua obtenidos sobre cualquiera de los 4 tubos de este árbol representan, para cualquier fecha, el contenido total medio, a la misma distancia de los otros árboles equipados, con una incertidumbre inferior al $5 \%$.

Resultados completamente análogos se han obtenido sobre A15, lo que ha conducido a considerar estos dos sitios como representativos, a la escala de las subparcelas, para el cálculo del balance hídrico.

\section{EJEMPLO METODOLOGICO}

\section{A. Balance hidrico}

\section{Método de ponderación de las medidas con sonda de neutrones}

Durante el año hidrológico, se observa que las medidas de los perfiles hídricos obtenidas para todos los tubos de acceso muestran generalmente dos tipos de situación, representados en la fig. 3 para el árbol B2 :

- Durante el período de invierno y de primavera (noviembre a abril), período de lluvias, los perfiles hídricos son prácticamente idénticos de un tubo a otro.

- Luego, durante el período de riego, sólo los tubos 1 y 2 están afectados por el aporte de agua localizado, con un gradiente importante humedad desde el árbol hacia el exterior. El aspecto tridimensional del flujo necesita entonces un análisis especial de las medidas de humedad para obtener el balance a la escala de una superficie de referencia (marco de plantación de 25 ó $49 \mathrm{~m}^{2}$.

Se ha considerado, pues, para el cálculo del balance hídrico, durante el período de riego, que existen en el marco de plantación dos zonas distintas :

- una zona afectada por el riego, que comprende los tubos 1 y 2 . La superficie, verificada mediante medidas 
tensiométricas, corresponde aproximadamente a la «sombra del árbol», es decir un círculo de $4 \mathrm{~m}$ de diámetro;

- una zona no afectada por el riego, que comprende los tubos 3 y/o 4 según la dimensión del marco de plantación, y donde esta superficie vale 12,5 ó $36,5 \mathrm{~m}^{2}$.

De acuerdo con el esquema dado en la fig. 2 , se ha supuesto que los perfiles hídricos medidos en el tubo 1 caracterizan la existencia de un cilindro de $1 \mathrm{~m}$ de radio centrado sobre el árbol, mientras que el tubo 2 (a $1,5 \mathrm{~m}$ ) pertenecería a un anillo de 1 y $2 \mathrm{~m}$ de radio. Si $\Delta \mathrm{S}_{1}$ y $\Delta \mathrm{S}_{2}$ son las variaciones de contenido total de agua (stocks) medidos entre dos fechas sobre estos tubos, sus valores son ponderados por referencia a las superficies respectivas y referidas a una variación $\Delta \mathrm{S}^{*}{ }_{\mathrm{i}}$ característica de la zona afectada por el riego (i) dada por la expresión :

$$
\Delta \mathrm{S}_{\mathrm{i}}^{*}=\frac{\Delta \mathrm{S}_{1}+3 \Delta \mathrm{S}_{2}}{4}
$$

La misma expresión se aplica para el drenage.

En la zona no afectada por el riego $\left(\mathrm{n}_{\mathrm{i}}\right)$ se ha considerado que las transferencias de agua en el suelo son esencialmente verticales. En el caso que existan dos tubos de acceso (marco de plantación $7 \times 7 \mathrm{~m}$ ) la variación de stock (o el drenaje) característico de esta zona vale entonces :

$$
\Delta \mathrm{S}_{\mathrm{ni}}^{*}=\frac{\Delta \mathrm{S}_{3}+\Delta \mathrm{S}_{4}}{2}
$$

como consecuencia de la pequeñas diferencias generalmente observadas entre las medidas de los tubos $3 \mathrm{y}$ 4 (ver fig. 6).

Está claro que en el caso del marco $5 \times 5 \mathrm{~m}$ $\Delta S^{*}{ }_{n i}=\Delta S_{3}$. Por otra parte, en régimen de lluvias donde $\Delta \mathrm{S}_{2}$ es muy próximo a $\Delta \mathrm{S}_{1}$ la ponderación dada por la ecuación (1) se convierte en una media aritmética.

El cálculo del balance hídrico a nivel de la superficie del marco de plantación exige una nueva ponderación.

De acuerdo con las dimensiones del marco de plantación, los aportes de agua, las variaciones de stocks y el drenaje han sido ponderados en relación a las superficies de las zonas afectadas y no afectadas por el riego, calculando la evapotranspiración real (ETR) mediante las fórmulas siguientes :

$\mathrm{ETR}=\mathrm{P}+\mathrm{a}\left(\mathrm{I}-\Delta \mathrm{S}_{\mathrm{i}}^{*}-\mathrm{D}_{\mathrm{i}}\right)-\mathrm{b}\left(\Delta \mathrm{S}_{\mathrm{ni}}^{*}+\mathrm{D}_{\mathrm{ni}}^{*}\right)-\mathrm{R}(3)$

\section{Donde :}

- Para el marco $5 \times 5 \mathrm{~m}$ (subparcela A, 3 tubos) : $\mathrm{a}=\mathrm{b}=0,5$.

- Para el marco $7 \times 7 \mathrm{~m}$ (subparcela B, 4 tubos) : $\mathrm{a}=14, \mathrm{~b}=34$

donde $\Delta \mathrm{S}^{*}{ }_{\mathrm{i}}, \mathrm{D}^{*}{ }_{\mathrm{i}}, \Delta \mathrm{S}^{*}{ }_{\mathrm{n} \text { i }}, \mathrm{D}^{*}{ }_{\mathrm{ni}}$ son los valores ponderados de las variaciones de stock y del drenaje, en las zonas afectada y no afectada por el riego, I es la dosis de riego por árbol, P la lluvia y R la escorrentía. Todos estos valores son característicos del intervalo de cálculo.
La ecuación (3) puede escribirse en una forma más general :

$$
\mathrm{ETR}=\mathrm{P}+\mathrm{I}^{*}-\Delta \mathrm{S}^{*}-\mathrm{D}^{*}-\mathrm{R}
$$

donde los valores con * han sido ponderados a la escala del marco de plantación.

El balance hídrico, determinado sobre una base de 15 días en general, se ha calculado para una capa de suelo desde $O$ hasta una profundidad de $2,4 \mathrm{~m}$ por ser ésta la máxima útil que alcanzan los tubos de acceso de la sonda de neutrones. El drenaje ha sido calculado aplicando la ley de Darcy y empleando las conductividades hidraúlicas obtenidas de acuerdo con el método que se expondrá a continuación.

Finalmente, la escorrentía $\mathrm{R}$, que sólo interviene durante las lluvias intensas del invierno y fuera de los períodos de riego, se ha estimado indirectamente suponiendo que en dicha época ETR es igual ETP Penman, según el método propuesto por VACHAUD y col. (1985b).

\section{Caracterización de la conductividad $k(\hat{E})$}

En 1983 se llevaron a cabo dos experiencias de caracterización hidrodinámica según el método de drenaje interno (HiLlel y col. 1972), en las proximidades de los árboles A15 y B2, cuyos resultados pueden verse en MoREno y col. (1983). Estas experiencias permitieron caracterizar entonces la capa de suelo situada entre la superficie y una profundidad máxima de $1,5 \mathrm{~m}$. En esta capa existen pocas diferencias entre los resultados obtenidos en cada uno de los casos, así como la ausencia de un efecto debido a la profundidad.

Una relación analítica de tipo :

$$
k=a \exp (b \theta)
$$

ha sido adoptada para cada uno de estos sitios. Del ajuste de los puntos experimentales se obtienen las curvas representadas en la fig. 4a, cuyos detalles se encuentra en el trabajo de Moreno y col. (1983).

Con el fin de evitar cualquier interferencia con la extracción radicular, el cálculo del drenaje se ha realizado a la produndidad de $2,4 \mathrm{~m}$, es decir, por debajo de la probable zona radicular activa. Para tratar de verificar las posibilidades de extrapolación de estas relaciones a dicha profundidad se efectuó un nuevo muestreo y análisis granulométrico. Los resultados (MORENO y col., 1987) han mostrado que el suelo en B2 permanece homogéneo hasta esa cota, mostrando la misma textura que en superficie. Por el contrario, en A15, los resultados muestran la existencia de una zona más limosa por debajo de $2 \mathrm{~m}$ de profundidad, donde probablemente hay una disminución de $\mathrm{k}$.

Para obtener una estimación de $\mathrm{k}(\theta)$ a la profundidad de $2,4 \mathrm{~m}$ se ha efectuado, durante los tres años, un cálculo simplificado, en las condiciones siguientes :

- Período lluvioso de inverno, con precipitacíon de débil intensidad (ausencia de escorrentía), de duracion 

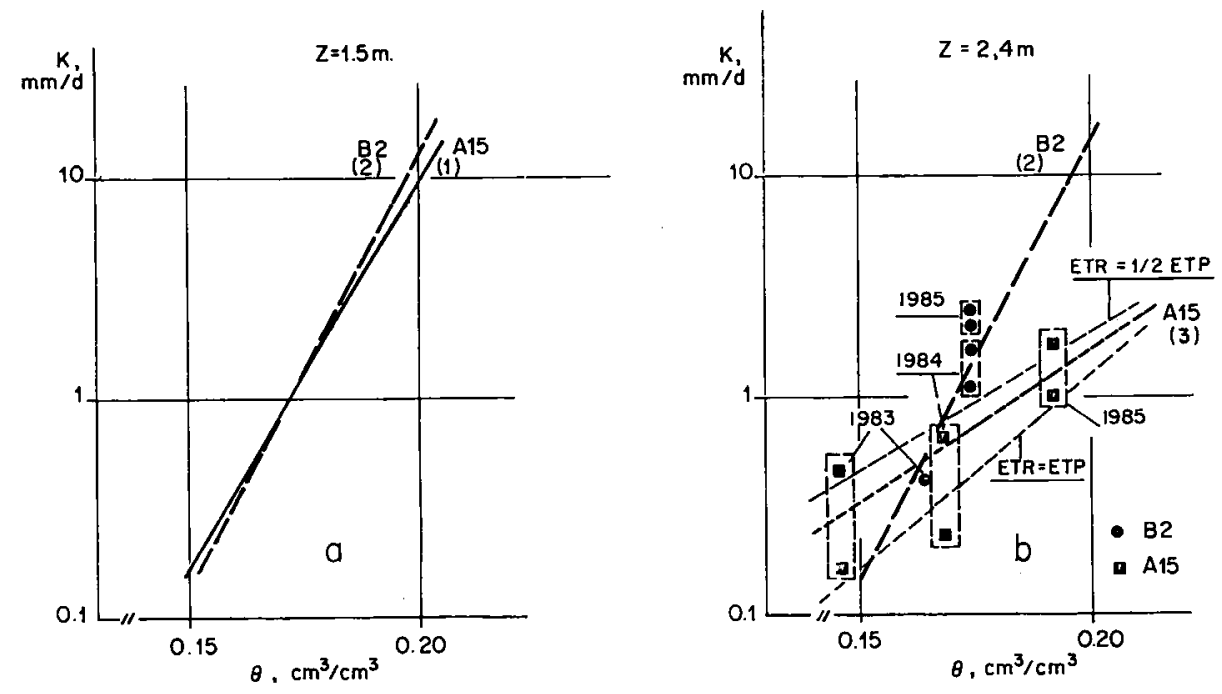

Figure 4

Curvas $K(\hat{E})$. Comparicion entre valores obtenidos por drenage interno entre 0-1.5 m (4-a) y por metodo simplificado a $2.4 \mathrm{~m}$, con valores de tabla 1 (4-b).

Hydraulic conductivity $K(\hat{\mathrm{E}})$. Comparison between values obtained at depth $1.5 \mathrm{~m}$ by internal drainage (4-a) and at depth $2.4 \mathrm{~m}$ using the simplified method summarized in table $1(4-b)$.

Conductivité hydraulique $K(\hat{E})$. Comparaison entre valeurs obtenues à $1,5 \mathrm{~m}$ par drainage interne (4-a) et à 2,4 m par la méthode du bilan simplifiée, d'après les valeurs données tableau I (4-b).

aproximada de un mes; contenido de agua $(\theta)$ y contenido total de agua (S) muy próximos en todos los tubos, a la profundidad de $2,4 \mathrm{~m}$; flujo de drenaje gravitatorio en la base del perfil.

Se ha supuesto, asimismo, que en este período ETR estaba comprendida entre 0,5 y 1 ETP Penman. Eajo estas condiciones, la conductividad hidraúlica viene dada directamente por la ecuación :

donde $\theta^{*}$ es el contenido de agua volumétrico equivalente al marco de plantación.

$$
k\left(\theta^{*}\right)=\left(\mathrm{P}-\Delta \mathrm{S}^{*}-\mathrm{ETR}\right) / \Delta \mathrm{t}
$$

En la tabla 1 se muestran, de forma resumida, los valores obtenidos en A15 y B2 para los tres años.
Es necesario señalar que $\mathrm{k}\left(\theta^{*}\right)$ se afecta poco por el método elegido para el cálculo de ETR, a consecuencia de los bajos valores de ETP Penman durante el período considerado (entre 0,5 y $1 \mathrm{~mm} /$ día) frente a los otros términos de la ecuación (6). Teniendo en cuenta la variación bastante sensible de $\theta^{*}$, a $z=2,4 \mathrm{~m}$, de un año a otro, se puede definir una relatión $\mathrm{k}\left(\theta^{*}\right)$ que cubre una amplia gama de medidas. Parece claro que para A15 los puntos así obtenidos difieren bastante de los caracterizados a $1,5 \mathrm{~m}$ por drenaje interno, mientras que en B2 los valores que se obtienen por este método concuerdan bien con la curva obtenida anteriormente.

Los parámetros de la relación $\mathrm{k}(\boldsymbol{\theta})$ (ec. 5) utilizados para calcular el flujo a la profundidad de $2,4 \mathrm{~m}$ son los siguientes, para $\mathrm{k}$ en $\mathrm{mm} / \mathrm{dí}$ y $\theta$ en $\mathrm{m}^{3} / \mathrm{m}^{3}$ :

TABLA 1

Estimacion de $K(\hat{\mathrm{E}})$ mediante el balance simplificado.

Estimation of $K(\hat{\mathrm{E}})$ by the simplified balance.

Estimation de $K(\hat{\mathrm{E}}) \grave{a}$ partir de la méthode simplifiée dddu bilan hydrique (eq. 7).

\begin{tabular}{|c|c|c|c|c|c|}
\hline \multirow{2}{*}{$\begin{array}{l}\text { Sitio } \\
\text { Site } \\
\text { Site }\end{array}$} & \multirow{2}{*}{$\begin{array}{l}\text { Periodo } \\
\text { Period } \\
\text { Période }\end{array}$} & \multirow{2}{*}{$\begin{array}{l}\theta^{*}\left(\mathrm{~m}^{3} / \mathrm{m}^{3}\right) \\
\mathrm{z}=2,4 \mathrm{~m}\end{array}$} & \multirow{2}{*}{$\sigma\left(\theta^{*}\right)$} & \multicolumn{2}{|c|}{$\mathrm{K}(\mathrm{mm} / \mathrm{d})$} \\
\hline & & & & $\mathrm{ETR}=\mathrm{ETP}$ & $\mathrm{ETR}=1 / 2 \mathrm{ETP}$ \\
\hline A 15 & $\begin{array}{l}09 / 11 / 82-24 / 01 / 83 \\
10 / 01 / 84-05 / 03 / 84 \\
31 / 01 / 85-20 / 03 / 85\end{array}$ & $\begin{array}{l}0,146 \\
0,168 \\
0,193\end{array}$ & $\begin{array}{l} \pm 0,001 \\
\pm 0,001 \\
\pm 0,005\end{array}$ & $\begin{array}{l}0,17 \\
0,23 \\
1,12\end{array}$ & $\begin{array}{l}0,48 \\
0,65 \\
1,80\end{array}$ \\
\hline B2 & $\begin{array}{l}14 / 12 / 82-21 / 02 / 83 \\
24 / 11 / 83-19 / 12 / 83 \\
31 / 01 / 85-20 / 03 / 85\end{array}$ & $\begin{array}{l}0,166 \\
0,173 \\
0,176\end{array}$ & $\begin{array}{l} \pm 0,005 \\
\pm 0,003 \\
\pm 0,005\end{array}$ & $\begin{array}{l}\overline{2,00} \\
1,72\end{array}$ & $\begin{array}{l}0,39 \\
2,40 \\
1,05\end{array}$ \\
\hline
\end{tabular}




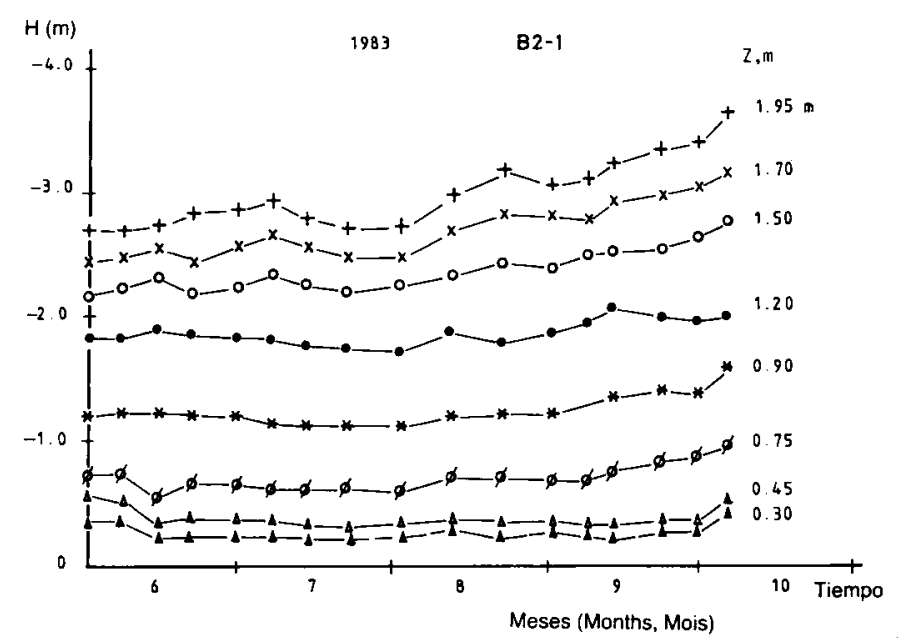

Figure 5

Evolucion de medidas tensiometricas en $B 2-I$ durante el periodo de riego de 1983 (carga hidraulica $H$.).

Changes of water head in B2-1, during the irrigation period, in 1983.

Evolution des mesures tensiométriques sur $B 2$ pendant la période d'irrigation 1983 (charge hydraulique $H$ ).

para

A15 $\quad \mathrm{a}=2,510 \cdot 10^{-3} ; \quad \mathrm{b}=32,327 \quad$ (curva 3 )

(obtenido por correlación visual entre los puntos de medida simplificada)

para

B2

$$
\mathrm{a}=4,490 \cdot 10^{-7}
$$

$\mathrm{b}=85,650 \quad($ curva 2$)$

\section{Ejemplo de aplicación B2 1983 y 1984}

Para ilustrar el método hemos considerado los resultados obtenidos durante los años hidrológicos 1983 y 1984 (nov. 1982 a nov. 1984) en el sitio B2 (0,4 EV del tanque y marco de plantación $7 \times 7 \mathrm{~m}$ ).

Esto años se han caracterizado por la sucesión de una pluviometría muy débil $(258,3 \mathrm{~mm})$, concentrada entre la primera década de noviembre y la primera de mayo, para el año hidrológico 1982-1983 y después fuerte $(542,7 \mathrm{~mm})$ entre finales de octubre de 1983 y finales de junio de 1984. El riego había comenzado el primero de mayo, finalizando hacia el 15 de octubre de 1983, mientras que en 1984 la fecha de comienzo fue a principios de junio y la de terminación a mediados de octubre. Cuatro series de perfiles hídricos característicos correspondientes, respectivamente, al momento inicial, antes de las lluvias de invierno (27/10/82), al final del período de lluvias $(28 / 04 / 83)$, durante el período de riego $(15 / 07 / 83)$ y al momento final $(26 / 10 / 83)$ se han mostrado en la fig. 3. Es necesario apuntar la gran uniformidad de los perfiles durante la época de las lluvias y unas diferencias muy netas entre los sitios durante el período de riego. Se dispone, para 1983, de un conjunto de 20 fechas de medida, que están recogidas en el anexo técnico (MORENo y col., 1987).

La evolución de las medidas tensiométricas sobre B2-1, iniciadas al comienzo del riego, están recogidas en la fig. 5. Dos informaciones pueden obtenerse de esta gráfica :

- En superficie, hasta $0,75 \mathrm{~m}$, el suelo está saturado durante todo el período de riego prácticamente.

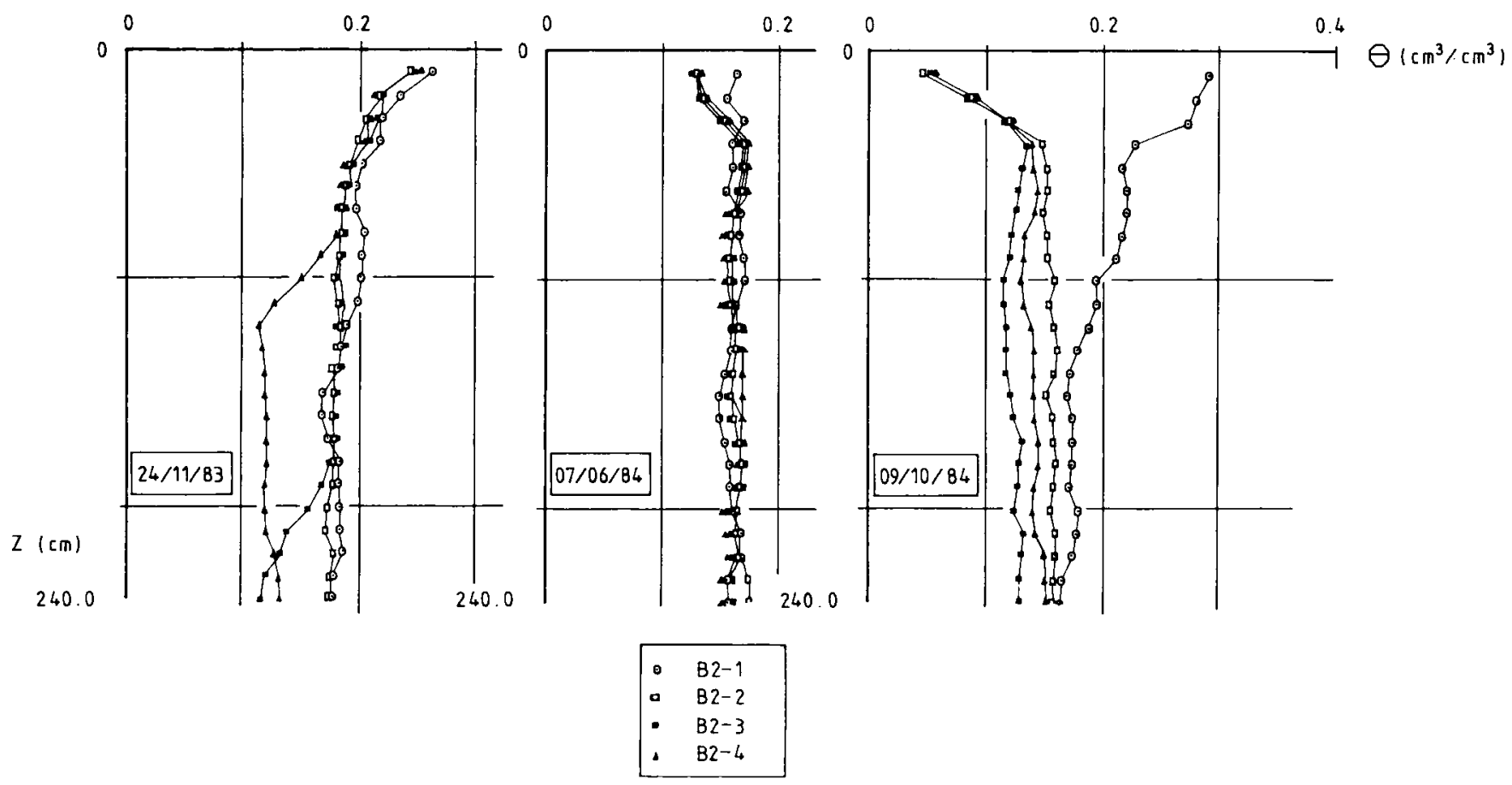

Figure 6

Perfiles hidricos en B2, en diversas fechas de 1983-84.

Water profiles in B2, at different dates in 1983-84.

Profils hydriques sur B2 à différentes dates en 1983-84. 

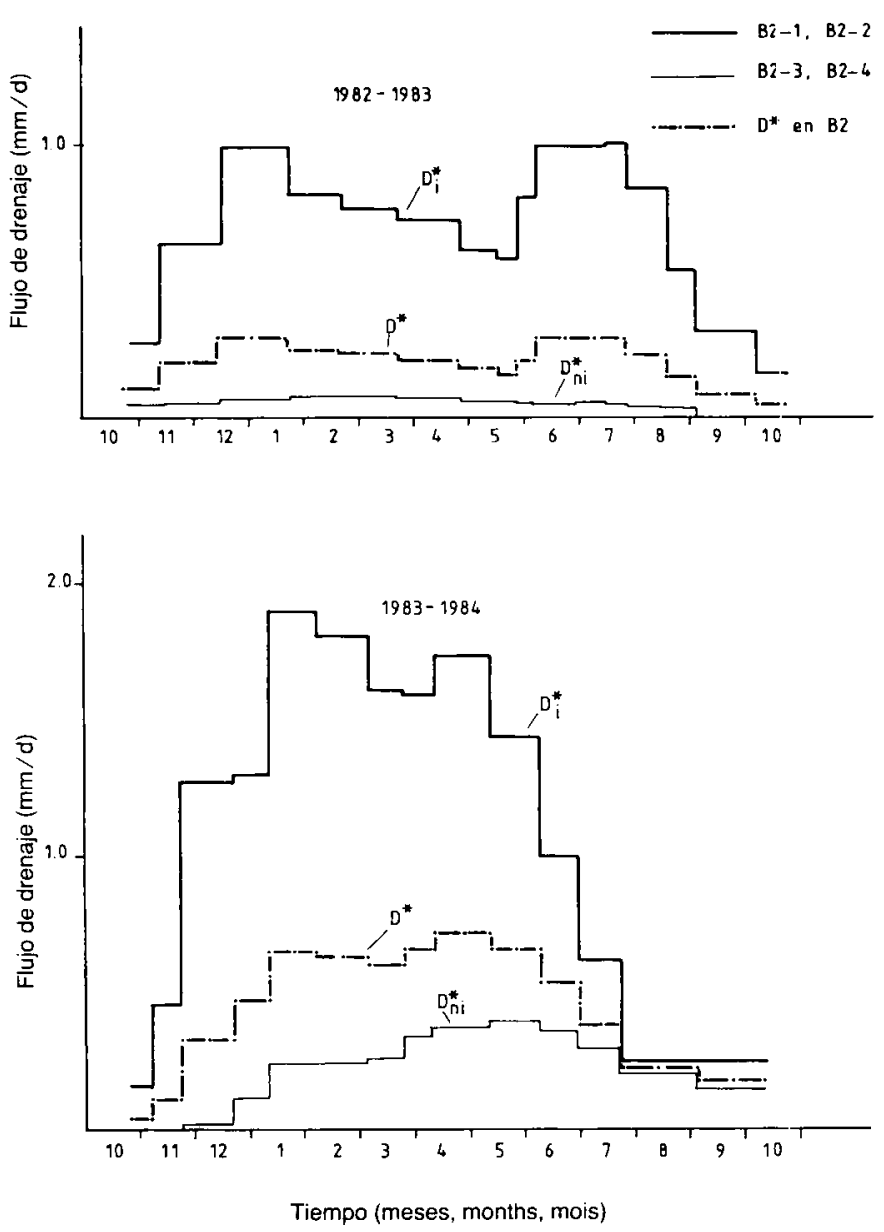

Figure 7

Evolucion del flujo de drenaje ponderado en B2 durante 1983 y 1994. Changes of weighted drainage fux in B2, during 1983 and 1984. Evolution du flux de drainage pondéré sur B2 en 1983-84.
- En profundidad (entre 1,5 y 2,0 m), el gradiente hidraúlico indica un drenaje importante. Se puede, por consiguiente, estimar (en este caso) que el aporte de agua por el riego es demasiado fuerte, aunque esté limitado el 0,4 de la evaporación medida en el tanque y referido a la sombra del árbol.

El año 1984, de hidrología media, se ha caracterizado por lluvias muy fuertes entre el 15 de octubre y el 15 noviembre $(220 \mathrm{~mm})$, que prácticamente representan el valor anual del año precedente. Teniendo en cuenta las fuertes diferencias entre los perfiles hídricos de los tubos B2-1 y B2-4 en octubre de 1983 (fig. 3), la humectación del perfil de suelo con estas lluvias es muy diferente. En la fig. 6 se encuentran representados los perfiles hídricos característicos medidos en noviembre de 1983, al comienzo del período de riego ( 7 de junio de 1984) y al final del periodo de riego ( 9 de octubre).

Haciendo el mismo análisis detallado que en el año anterior, los resultados más importantes obtenidos son los siguientes :

- Durante el período de invierno, y a consecuencia de las fuertes lluvias, hay un drenaje en todos los tubos (aumento del contenido de agua a 2,4 $\mathrm{m}$ de profundidad y presencia de un gradiente de carga hidraúlica próximo $\mathrm{a}-1)$.

- En el período de riego, los contenidos de agua y el gradiente observado en la base de todos los tubos permanecen en niveles suficientemente elevados para que exista drenaje. Por otra parte, en el tubo B2-1, como en el caso anterior, el suelo permaneció saturado en superficie.

Teniendo en cuenta la relativamente elevada incertidumbre de $\mathrm{k}(\theta)$, el drenaje ha sido calculado sobre la hipótesis de un flujo gravitatorio. Este cálculo se ha realizado, tubo por tubo, durante los dos años considerados, aplicando la ley de Darcy a la profundidad de $2,4 \mathrm{~m}$ y utilizando los coeficientes de la ecuación (7)
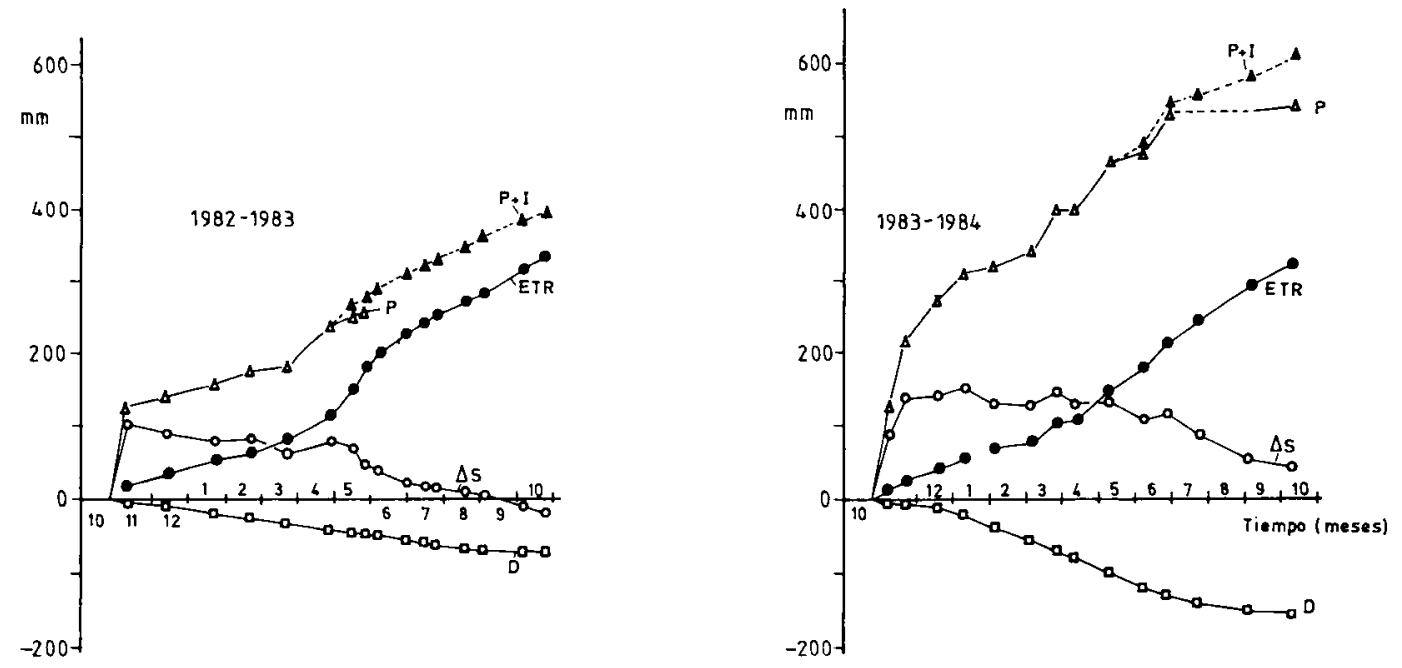

Figure 8

Evolucion anual de los parametros (acumulados) del balance hidrico en B2 para 1983 y 1984.

Annual changes of water balance (cumulated) parameters, in B2, during 1983 and 1984.

Evolution annuelle des paramètres cumulés du bilan hydrique sur B2 en 1983 et 1984. 
para B2. Para cada intervalo de medida, se ha considerado el contenido de agua medio $(\theta)$ durante el período. El detalle de los resultados está recogido por MORENO y col. (1987) ; los valores de los flujos de drenaje ponderados sobre el conjunto del marco $\left(D^{*}\right)$ y en las zonas regadas y no regadas se han representado en la fig. 7 . Esta estimación del drenaje $\mathrm{D}^{*}$, así como las medidas de lluvia $(P)$, riego $\left(I^{*}\right)$, variación de stock $\left(\Delta S^{*}\right)$, ponderados sobre el marco de planación, se hallan recogidas en la fig. 8 en forma acumulada. La escorrentía tiene lugar solamente en noviembre de 1983, como consecuencia de las lluvias intensas.

Para los dos años expuestos, el balance referido a la superficie del marco de plantación $\left(49 \mathrm{~m}^{2}\right)$ muestra los valores siguientes:

- En 1983, ETR es igual a $337,4 \mathrm{~mm}$, para una demanda evaporativa de $966 \mathrm{~mm}$ y un aporte de agua (lluvia + riego) de $394,1 \mathrm{~mm}$, siendo el drenaje $72,7 \mathrm{~mm}$.

- En 1984, la evapotranspiración alcanza los $324,4 \mathrm{~mm}$, para una demanda evaporativa de $836,9 \mathrm{~mm}$, un aporte de agua de $607,5 \mathrm{~mm}$ (lluvia + riego) y un drenaje de $155,5 \mathrm{~mm}$.

\section{RESULTADOS DE CUATRO AÑOS DE EXPERIENCIAS}

\section{A. Estudio interanual en las parcelas}

El estudio metodológico realizado en B2 indica especialmente que, en estos dos años sucesivos, la zona afectada por el riego se encuentra siempre en un nivel de humedad elevado, lo cual ocasiona un drenaje importante.

La comparación de los perfiles hídricos obtenidos, cada año, en los tubos B2-1 y B2-4, a partir de 1981, en dos fechas características, al comienzo del riego (mayojunio) y al final del verano (octubre), es particularmente interesante, como puede verse en la fig. 9. Esta figura muestra, en efecto, que en el punto B2-1, el suelo está cada vez más húmedo al comienzo del período de riego (excepto en 1983, caracterizado por una pluviometría muy débil) y al final del verano. Por el contrario, en B2-4, los perfiles hídricos (correspondientes a la situación natural) son todos muy similares de un año a otro, excepto en 1983, donde el suelo está mucho más seco. Es preciso hacer notar que los perfiles hídricos de partida en B2-1 y B2-4 son idénticos.

Estos resultados son aún más claros cuando se considera el stock de agua del suelo. En la fig. 10 se muestra la evolución de la variación de stock, en la capa de suelo $0-2,40 \mathrm{~m}$, referida al perfil hídrico medido el $27 / 10 / 81$, hasta octubre de 1986 (las medidas realizadas en 1981 no han sido lo suficientemente próximas entre sí en el tiempo para tenerlas en cuenta a nivel de la evolución). Mientras que en el sitio B2-4 se observa una variación anual caracterizada por un incremento ligado a la pluviometría y después por una disminución debida al agotamiento de la reserva del suelo, que supone llegar, en cada año, a una situación muy próxima a la de partida, en el B2-1 se ve claramente una tendencia interanual al crecimiento del stock de agua del suelo, el cual supone un incremento de $140 \mathrm{~mm}$ en los cinco años de experimentación.

Finalmente, se ha completado el cálculo del balance hídrico, para B2, considerando todos los años. Los valores, reagrupados en la tabla 2 , se analizarán más adelante.

El mismo análisis que en el caso precedente se ha realizado en este tratamiento considerando el sitio A 15 . De manera análoga, como en $\mathrm{B} 2$, se observa que el perfil hídrico, próximo al gotero, está más húmedo cada año. Por el contrario, el perfil correspondiente a la zona seca es muy similar de un año a otro. Esta observación explica la evolución de la variación de stock, en la capa de suelo $0-2,40 \mathrm{~m}$, referida al perfil medido el $27 / 10 / 81$ y que se muestra en la Fig. 11 para cada uno de los dos tubos mencionados.

En resumen, la variación interanual del contenido total de agua (stock) a partir del 27/10/81 (Fig. 11) muestra una tendencia clara a un incremento muy importante en A15 (alrededor de $200 \mathrm{~mm}$ en 4 años), mientras que en las zonas no afectadas por el riego se vuelve cada año a una situación muy similar a la de partida.

Es necesario apuntar que los aumentos de stock medidos en A15 (lo mismo en A15-1 que en A15-3) son notablemente más importantes que en $\mathrm{B} 2$, lo que probablemente esté relacionado con la presencia, en profundidad, de la zona más limosa. Los valores de los términos del balance en A15, están igualmente recogidos en la tabla 2.

\section{B. Comparacion entre tratamientos y efecto sobre la produccion}

Con el método utilizado inicialmente para la determinación de la dosis de riego (aporte referido a la superficie de la sombra del árbol) la diferencia en el marco de plantación induce eventualmente, en términos del balance hídrico de la superficie del marco o de la plantación, a un efecto de disminución de la dosis real de riego cuando se pasa de un tratamiento de alta densidad de plantas a otro de baja densidad.

En el estudio realizado, se llega, en último término, a comparar un tratamiento con una dosis de riego ponderada de $0,2 \mathrm{EV}$ (marco de plantacion $5 \times 5 \mathrm{~m}$, ec. (3)) con un tratamiento cuya dosis de riego ponderado es de $0, I E V$ (marco de plantación $7 \times 7 \mathrm{~m}$, ec. (3)) cuando se refiere a superficie plantada y no a un árbol individual. Estos valores se obtienen al multiplicar por $1 / 2$ y $1 / 4$ (relaciones sombra del árbol-superficie marco de plantación, relativas a $5 \times 5 \mathrm{~m}$ y $7 \times 7 \mathrm{~m}$ respectivamente) el coeficiente de reducción $0,4 \mathrm{EV}$.

Todos los resultados del balance se muestran de forma globalizada en la tabla 2. Con el fin de precisar el análisis de la eficiencia del riego se han considerado los valores globales de los diferentes componentes del balance, a escala del marco de plantación, durante dos períodos cada año :

- El período sin riego, correspondiente a las lluvias de invierno.

- El período de riego (28/04 al 26/10 en $1983 ; 11 / 05$ al $09 / 10$ en $1984 ; 20 / 03$ al $07 / 10$ en 1985 y $01 / 05$ al $14 / 10$ en 1986). 

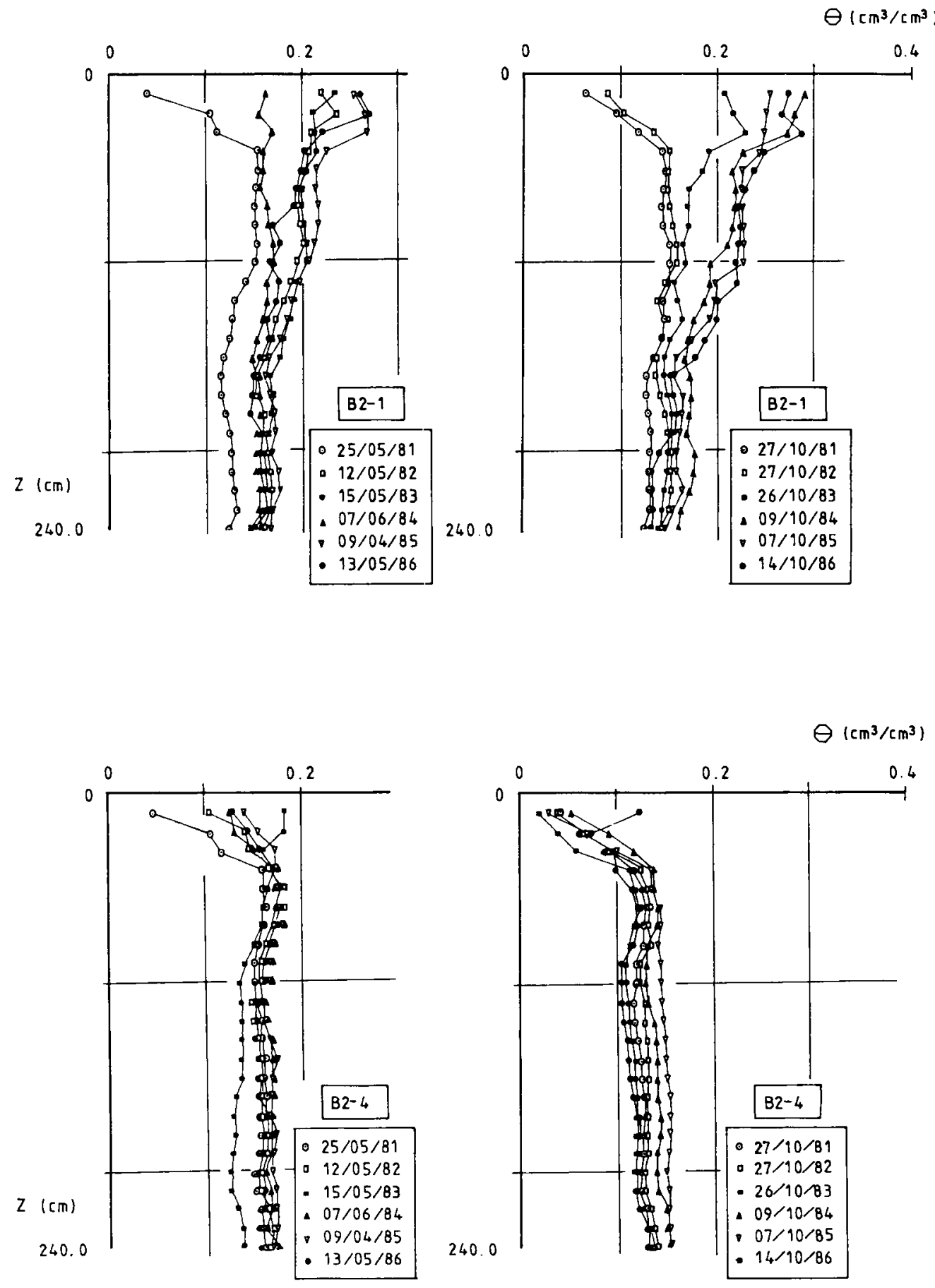

Figure 9

Perfiles hidricos en B2-I y B2-4, al comienzo del riego y al final del verano de 1981 a 1986.

Water profiles in B2-I and B2-4, at the beginning and end of the irrigation period from 1981 to 1986.

Profils hydriques sur B2.1 et B2.4 au début et à la fin des périodes d'irrigation de 1981 à 1986.

El análisis de los datos de esta tabla conduce a las siguientes consideraciones :

\section{Drenaje}

- Existe una relación estrecha entre drenaje y pluviometría durante el período invernal, cuando el drenaje es más importante, exceptuando el año 1985 en el tratamiento $5 \times 5 \mathrm{~m}$, en el que existe también un drenaje bastante considerable en verano, causado por las cuantiosas lluvias de primavera y por el nivel de riego al mismo tiempo.
- El drenaje es sistemáticamente más importante en el tratamiento $5 \times 5 \mathrm{~m}$. Ello es debido principalmente a la considerable extensión de la zona afectada por el riego : la mitad de la superficie del marco frente a una cuarta parte en el caso del $7 \times 7 \mathrm{~m}$, y a un contenido de humedad del suelo más elevado al comienzo del invierno. Ello supone que al final del verano, en el marco $5 \times 5 \mathrm{~m}$, el perfil debe tener un contenido de agua suficiente que permita que el drenaje comience de inmediato, en la mayor parte de la superficie, una vez recibidas las primeras lluvias. Por el contrario, éste no 

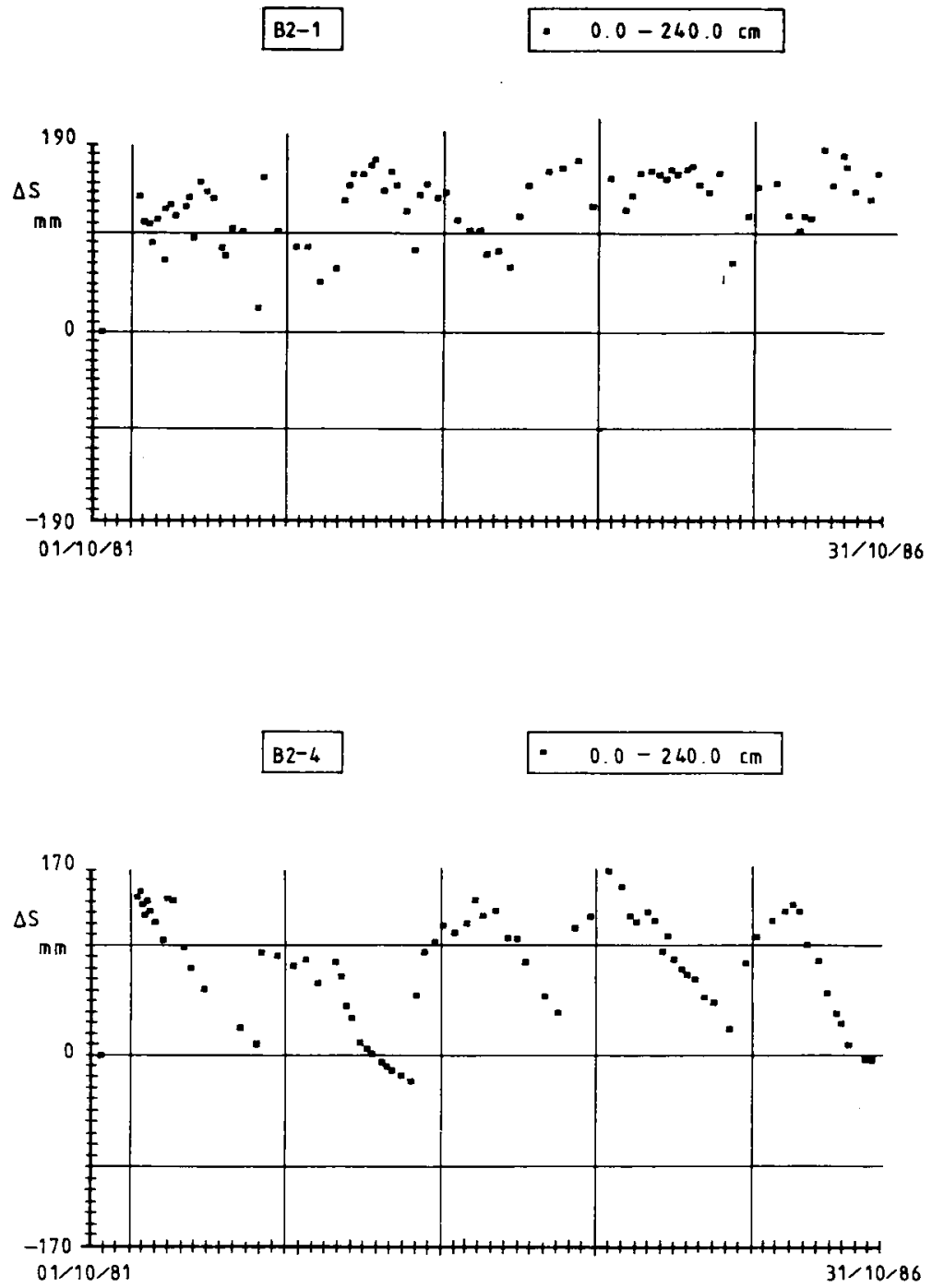

Figure 10

Evolucion comparada del stock para la capa de suelo de 0 a 2,4 m en B2-1 y B2-4, a partir del perfil hidrico de 27/10/81 hasta octubre de 1986. Compared changes of water storage profiles, between 0 and $2.4 \mathrm{~m}$ depth, in B2-1 and B2-4, from 27/10/81 to October 1986.

Evolution comparée des variations de stock, dans la tranche 0-2,4 m sur B2-1 et B2-4, d'octobre 1981 à octobre 1986.

debe ser el caso del marco $7 \times 7 \mathrm{~m}$, donde el stock de agua debe reconstituirse con las primeras lluvias antes de que el conjunto del marco permita el drenaje. De esta forma, no es raro que también en invierno el drenaje sea más importante en el marco $5 \times 5 \mathrm{~m}$.

\section{Riego}

- En un año seco (1983), el riego es relativamente eficaz, puesto que las pérdidas por drenaje durante el período que comprende representan globalmente sólo alrededor del $20 \%$ del agua aportada, en los dos marcos de plantación. En los dos años más húmedos (1984 y 1985), por el contrario, en tres de cuatro casos, las pérdidas por drenaje son equivalentes a los aportes por riego.

Por otra parte, el aporte de agua equivalente al coeficiente $0,4 \mathrm{EV}$ y referido a la sombra del árbol, parece ser el más adecuado a plantaciones con una densidad superior a 200 árboles/ha, como indica LE BouRdELLEs (1986). Asimismo, las cantidades de agua aportadas en este estudio son bastante similares a las encontradas como más adecuadas por CRUZ-CONDE y FUENTES (1986) para una producción óptima, ya que por encima de este nivel de aporte incluso decrece la producción.

\section{Consumo}

Las diferencias entre tratamientos son menos importantes en lo que a consumo de agua se refiere. No obstante, es necesario diferenciar los dos períodos.

En invierno, las diferencias entre tratamientos son demasiado pequeñas como para que puedan ser consideradas como significativas. Es necesario mencionar que la ETR acumulada de este período, donde la demanda evaporativa es particularmente débil, está comprendida entre 0,5 y 0,6 ETP Penman, para los años $82-83$ y $83-84$, entre 0,7 y 0,9 , para el año $1984-85$, y alrededor de 0,7 en el año 1985-86.

Durante el período de riego, por el contrario, la ETR es sistemáticamente más elevada en el marco de planta- 
TABLA 2

Valores totales de lluvia $(P)$, riego $(I)$, drenaje ponderado (D), evapotranspiracion real (ETR) y evapoiranspiracion potencial (ETP), durante el periodo de lluvias y el de riego.

Total values of precipitation ( $P$ ), irrigation (I), weighted drainage (D), actual evapotranspiration (ETR), and potential evapotranspiration (ETP), during precipitation and irrigation periods.

Valeurs cumulées de la pluie (P), de l'irrigation (I), du drainage ípondéré) (D) de l'évapotranspiration réelle (ETR) et potentielle (ETP) durant les périodes d'hiver puis d'irrigation.

\begin{tabular}{|c|c|c|c|c|c|c|c|}
\hline $\begin{array}{l}\text { Ano hidrologico } \\
\text { Hydrological year } \\
\text { Année hydrologique }\end{array}$ & $\begin{array}{l}\text { Periodo } \\
\text { Period } \\
\text { Période }\end{array}$ & $\underset{(\mathrm{mm})}{\mathrm{P}}$ & $\begin{array}{c}\mathrm{I} \\
(\mathrm{mm})\end{array}$ & $\underset{(\mathrm{mm})}{\mathrm{D}}$ & $\underset{(\mathrm{mm})}{\mathrm{R}}$ & $\begin{array}{c}\text { ETR } \\
(\mathrm{mm})\end{array}$ & $\begin{array}{c}\text { ETP } \\
(\mathrm{mm})\end{array}$ \\
\hline \multicolumn{8}{|c|}{$\begin{array}{l}\text { Marco de plantacion } 7 \times 7 \mathrm{~m} \text {. Riego ponderado : } 0,1 \mathrm{EV} \\
\text { Plant spacing } 7 \times 7 \mathrm{~m} \text {. Weighted irrigation : } 0.1 \mathrm{EV} \\
\text { Distance de plantation } 7 \times 7 \mathrm{~m} \text {. Apport d'irrigation pondéré sur la maille : } 0,1 \mathrm{EV}\end{array}$} \\
\hline 1983-1984 & $\begin{array}{l}26 / 10 / 83 \\
11 / 05 / 84 \\
11 / 05 / 84 \\
09 / 10 / 84\end{array}$ & $\begin{array}{r}467,7 \\
74,7\end{array}$ & 65,1 & 101,7 & 81,7 & $\begin{array}{l}148,0 \\
174,4\end{array}$ & $\begin{array}{l}229,8 \\
607,1\end{array}$ \\
\hline $1985-1986$ & $\begin{array}{l}07 / 10 / 85 \\
26 / 04 / 86 \\
26 / 04 / 86 \\
14 / 10 / 86\end{array}$ & $\begin{array}{r}407,4 \\
30,9\end{array}$ & 100,5 & 51,4 & 95,6 & 167,7 & $\begin{array}{l}252,0 \\
729,7\end{array}$ \\
\hline \multicolumn{8}{|c|}{$\begin{array}{l}\text { Marco de plantacion } 5 \times 5 \mathrm{~m} \text {. Riego ponderado : } 0,2 \mathrm{EV} \\
\text { Plant spacing } 5 \times 5 \mathrm{~m} \text {. Weighted irrigation : } 0.2 \mathrm{EV} \\
\text { Distance de plantation } 5 \times 5 \mathrm{~m} \text {. Apport d'irrigation pondéré sur la maille : } 0,2 \mathrm{EV}\end{array}$} \\
\hline $1982-1983$ & $\begin{array}{l}21 / 09 / 82 \\
02 / 05 / 83 \\
02 / 05 / 83 \\
26 / 10 / 83\end{array}$ & $\begin{array}{r}244,5 \\
13,8\end{array}$ & 268,2 & $\begin{array}{l}75,0 \\
54,0\end{array}$ & & $\begin{array}{l}128,9 \\
296,1\end{array}$ & $\begin{array}{l}295,6 \\
699,4\end{array}$ \\
\hline 1984-1985 & $\begin{array}{l}09 / 10 / 84 \\
20 / 03 / 85 \\
20 / 03 / 85 \\
07 / 10 / 85\end{array}$ & 429,7 & 172,1 & 182,6 & 12,5 & 249,0 & 159,3 \\
\hline 1985-1986 & $\begin{array}{l}07 / 10 / 85 \\
26 / 04 / 86 \\
26 / 04 / 86 \\
16 / 10 / 86\end{array}$ & 407,4 & 200,1 & $\begin{array}{l}57,0 \\
87,5\end{array}$ & 84,5 & 257,1 & 732,7 \\
\hline
\end{tabular}

ción $5 \times 5 \mathrm{~m}$, lo que está de acuerdo con un contenido de agua del suelo más elevado en este tratamiento.

$\mathrm{La}$ identidad de los valores invernales nos parece ser una buena validación del método.

Es interesante observar, en los cálculos detallados del balance, que en cada año existe un período en el que se obtienen valores extremadamente bajos de ETR (Febrero, 1983 y 1984, Marzo, 1985). Este hecho debe estar asociado, probablemente, con la poda que sufren los árboles en este período, lo que daría lugar a una actividad de las plantas muy reducida.

\section{Relación con la producción}

Finalmente, se ha tratado de establecer una relación entre las observaciones de tipo hidrológico y la produc- cíon. Esta aproximación, debe ser considerada por el momento como un primer intento, ya que, por una parte, conocemos mal el tiempo de respuesta de un árbol como el olivo a una modificación drástica del manejo, con riego y fertilización, y por otra parte, el período de observación de la producción ha estado afectado por dos fenómenos naturales como las heladas tardías de 1983 y la alternancia en 1985. Se dispone de datos útiles de producción para 1981, 1982, 1984, y 1986, los cuales se muestran en la tabla 3 en forma de producción media (kg/árbol) y en función del marco de plantación, riego y fertilización. Sólo las producciones obtenidas en 1984 y 1986 podrían, de hecho, estar relacionadas con el presente estudio. Se incluyen, sin embargo, todos estos valores porque permiten ver claramente que no existe 

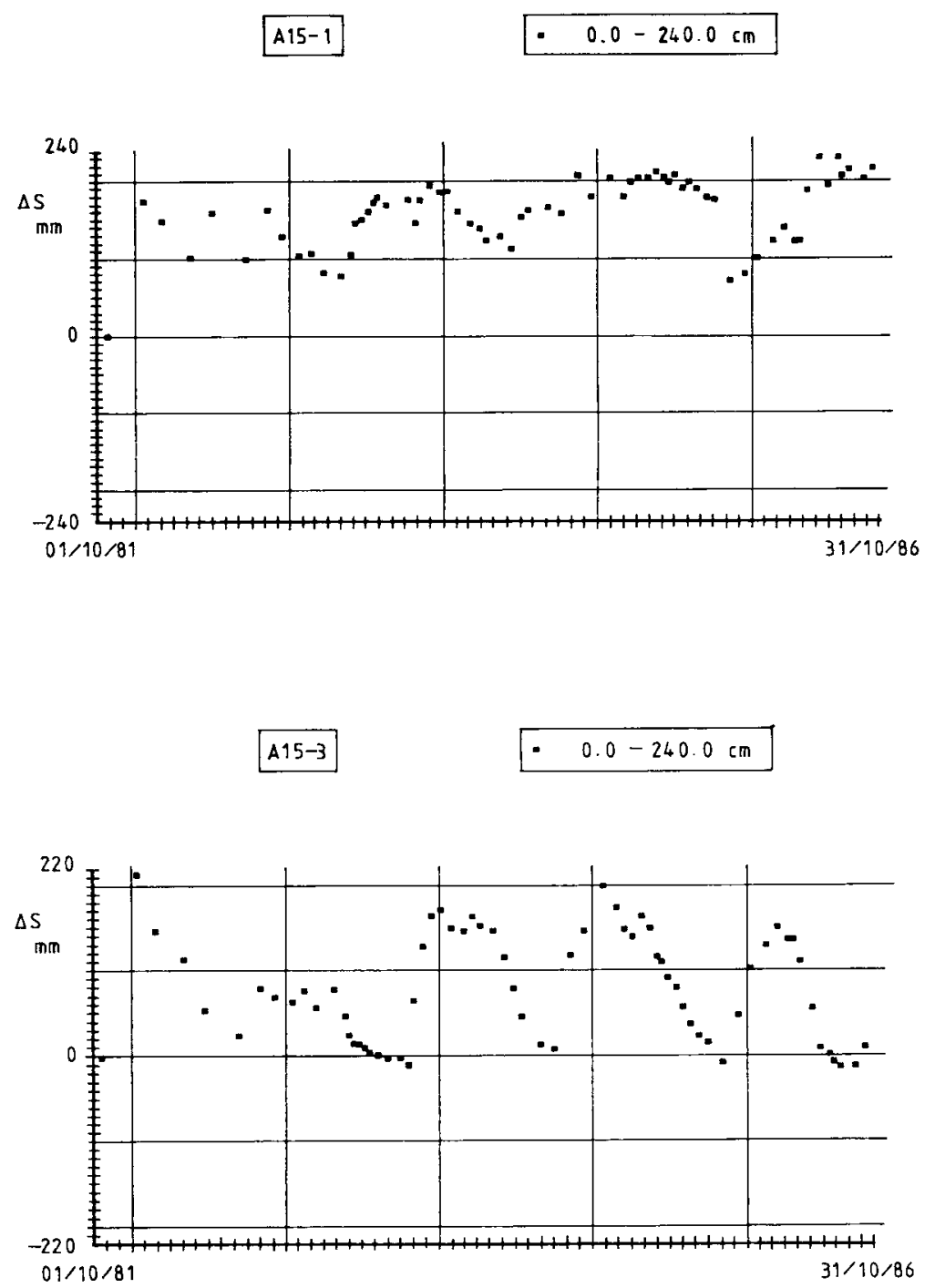

Figure 11

Evolucion comparada de la variacion de stock para la capa de suelo de 0 à 2,4 man A15-1 y A15-3, a partir del perfile hidrico de 27/10/81 hasta octubre de 1986.

Compared changes of water storage profiles between 0 and $2.4 \mathrm{~m}$ depth, in A15-I and A15-3, from 27/10/81 to October 1986.

Evolution comparée des variations de stock, dans la tranche 0-2,4 m, sur A15-1 et A15-3, d'octobre 1981 à octobre 1986.

ninguna diferencia significativa, a nivel del $5 \%$, durante estos cuatro años, entre dosis de riego, fertilización y producción. En uno de los bloques, la producción presenta valores sistemáticamente más bajos. Este bloque está situado en la parte más elevada de la parcela donde el suelo es más calcáreo.

La ausencia de efecto del riego debería ser achacada a la importancia de las pérdidas de agua (y de elementos fertilizantes) y de la elevada humedad del suelo en la zona radicular en las proximidades del gotero. Por otra parte, es necesario considerar que las escasas diferencias de ETR, de un año a otro, en cualquiera de los marcos de plantación podrían estar relacionadas con la forma de poda de los árboles de la parcela, la cual puede ser excesivamente drástica.

\section{CONCLUSIONES}

Al término de este análisis, el elemento esencial que resalta de este estudio parece ser el siguiente : el riego, al mismo tiempo demasiado fuerte e impropiamente aplicado, induce a pérdidas considerables de agua y fertilizantes.

Recordemos que se trataría, en principio, de aportar una respuesta práctica sobre la eficacia de los métodos. Por ello es posible actualmente establecer los siguientes puntos :

- El riego no debe programarse solamente en función de la demanda climática, sino de forma más fundamental en función de la humedad del suelo. El riego aplicado en una situación en la que el perfil hídrico posee un contenido de humedad elevado produce automáticamente pérdidas de agua importantes.

- El modo de riego, incluso, debe ser reconsiderado ya que el aporte continuado mediante los goteros crea un dominio de suelo saturado en la zona radicular, como se ha mostrado de forma clara.

Es necesario señalar que estos resultados han sido obtenidos con dosis de riego relativamente débiles $(0,1$ y $0,2 \mathrm{EV})$. Entre agricultores, las dosis empleadas pueden 
TABLA 3

Producción anual en función de las dosis de riego y fertilización. Valor medio por tratamiento ( $\mathrm{kg} /$ árbol). Annual yield in relation to water applied and fertilization. Mean value per treatment ( $\mathrm{kg} / \mathrm{tree})$.

Rendement annuel en relation avec l'apport d'eau et la fertilisation. Valeur moyenne par traitement ( $k g$ d'olives/arbre).

\begin{tabular}{|c|c|c|c|c|c|c|c|c|c|c|c|c|c|c|c|c|}
\hline \multirow{4}{*}{$\begin{array}{l}\text { Marco de } \\
\text { plantación } \\
\text { Plant spacing } \\
\text { Distance de } \\
\text { plantation } \\
5 \times 5 \mathrm{~m}\end{array}$} & \multicolumn{16}{|c|}{$\begin{array}{c}\text { Año } \\
\text { Year } \\
\text { Année }\end{array}$} \\
\hline & \multicolumn{4}{|c|}{$\begin{array}{c}1981 \\
\text { Riego } \\
\text { Irrigation }\end{array}$} & \multicolumn{4}{|c|}{$\begin{array}{c}1982 \\
\text { Riego } \\
\text { Irrigation }\end{array}$} & \multirow{2}{*}{\multicolumn{4}{|c|}{$\begin{array}{c}1984 \\
\text { Riego } \\
\text { Irrigation } \\
0,4 \mathrm{EV}\end{array}$}} & \multirow{2}{*}{\multicolumn{4}{|c|}{$\begin{array}{c}1986 \\
\text { Riego } \\
\text { Irrigation } \\
0,4 \mathrm{EV}\end{array}$}} \\
\hline & \multicolumn{2}{|c|}{$0,8 \mathrm{EV}$} & \multicolumn{2}{|c|}{$0,4 \mathrm{EV}$} & \multicolumn{2}{|c|}{$0,8 \mathrm{EV}$} & \multicolumn{2}{|c|}{$0,4 \mathrm{EV}$} & & & & & & & & \\
\hline & $\begin{array}{r}6,1 \\
\pm 10,1\end{array}$ & $\begin{array}{r}14,4 \\
\pm 8,5\end{array}$ & $\begin{array}{r}9,2 \\
\pm 6,3\end{array}$ & $\begin{array}{r}6,8 \\
\pm 6,0\end{array}$ & $\begin{array}{r}10,3 \\
\pm 11,2\end{array}$ & $\begin{array}{r}21,7 \\
\pm 7,6\end{array}$ & $\begin{array}{r}20,9 \\
\pm 9,5\end{array}$ & $\begin{array}{r}17,6 \\
\pm 18,8\end{array}$ & $\begin{array}{r}21,7 \\
+15,6\end{array}$ & $\begin{array}{r}39,5 \\
\pm 9,2\end{array}$ & $\begin{array}{l}38,3 \\
14,6\end{array}$ & $\begin{array}{r}36,7 \\
\pm 12,8\end{array}$ & $\begin{array}{r}25,7 \\
\pm 8,1\end{array}$ & $\begin{array}{r}27,5 \\
\pm 8,5\end{array}$ & $\begin{array}{r}27,7 \\
\pm 7,8\end{array}$ & 28,3 \\
\hline $7 \times 7 \mathrm{~m}$ & $\begin{array}{r}20,1 \\
\pm 8,2\end{array}$ & $\begin{array}{r}14,5 \\
\pm 8,7\end{array}$ & $\begin{array}{r}12,6 \\
\pm 7,2\end{array}$ & $\begin{array}{r}16,9 \\
\pm 9,0\end{array}$ & $\begin{array}{r}18,9 \\
\pm 7,9\end{array}$ & $\begin{array}{r}20,0 \\
+11,9\end{array}$ & $\begin{array}{r}20,9 \\
\pm 12,0\end{array}$ & $\begin{array}{r}14,1 \\
\pm 8,8\end{array}$ & $\begin{array}{r}44,9 \\
\pm 15,6\end{array}$ & $\begin{array}{r}36,5 \\
\pm 16,5\end{array}$ & $\begin{array}{r}37,7 \\
\pm 15,3\end{array}$ & $\begin{array}{r}44,0 \\
+12,9\end{array}$ & $\begin{array}{r}26,8 \\
+11,9\end{array}$ & $\begin{array}{r}28,3 \\
\pm 9,1\end{array}$ & $\begin{array}{r}29,4 \\
+12,0\end{array}$ & $\begin{array}{r}33,2 \\
\pm 7,8\end{array}$ \\
\hline $\begin{array}{l}\text { Fertilización } \\
\text { Fertilization } \\
\text { Fertilisation }\end{array}$ & $\mathrm{F} 1$ & $2 \mathrm{~F} 1$ & $2 \mathrm{Fl}$ & F1 & $\mathrm{Fl}$ & $2 \mathrm{Fl}$ & $2 \mathrm{Fl}$ & F1 & F0 & F1 & $2 \mathrm{~F} 1$ & $3 \mathrm{~F} 1$ & Fo & $\mathrm{F} 1$ & $2 \mathrm{~F} 1$ & $3 \mathrm{~F} 1$ \\
\hline
\end{tabular}

$\begin{aligned} \mathrm{F} 1= & 0,6 \mathrm{~kg} \text { nitrogeno, nitrogen, azote } & & \text { por arbol } \\ & 0.05 \mathrm{~kg} \text { fosforo, phosphorus, } & & \\ & \text { phosphore } & & \text { per tree } \\ & 0,35 \mathrm{~kg} \text { potasio, potassium } & & \text { par arbre }\end{aligned}$

$\mathrm{F} 0=$ fertilizacion cero, no fertilization, pas d'engrais.

ser mucho más elevadas. Por tanto, parece evidente que se ha realizado una economía de agua sustancial, a pesar de las pérdidas por drenaje.

En relación a la metodología, además del aspecto que ha permitido mostrar la importancia de la caracterización de la representabilidad espacial de los sitios de medidas, que ha sido ampliamente expuesto en la primera parte del estudio, se han podido definir los límites de aplicación del método de Darcy para caracterizar el flujo de drenaje.

Se ha mencionado ya la incertidumbre del cálculo de $\mathrm{k}$ para contenidos de agua elevados, lo que ha conducido a sugerir identificar ETR con ETP, durante el período de lluvias de invierno, para calcular el balance.

Otra precaución importante en la aplicación de la ley de Darcy hallada en este estudio, y más generalmente en otros estudios que tienen en cuento la vegetación, consiste en asegurarse que no existe extracción de agua por las raíces por debajo de la profundidad elegida para calcular el drenaje. En nuestro caso, la profundidad utilizada de $2,40 \mathrm{~m}$, parece suficiente (como se observó en un estudio preliminar efectuado sobre esta parcela por NuÑEZ y col. (1981), utilizando el método de la trinchera). En otro trabajo en curso, más reciente, que ha empleado el marcaje con $\mathrm{P}^{32}$, los primeros resultados (FERNANDEZ y col., 1987) muestran que, en las parcelas regadas, la respuesta al $\mathrm{P}^{32}$ se encuentra esencialmente en un volumen de suelo de una profundidad y radio de $1 \mathrm{~m}$ aproximadamente alrededor del árbol. Estas medidas sugieren, asimismo, que la actividad de las raíces por debajo de una profundidad de $2,40 \mathrm{~m}$ ó a una distancia de más de $2,5 \mathrm{~m}$ del árbol, durante el período de riego, debe ser muy reducida.

La transferencia hídrica en el suelo, alrededor del árbol, presenta una característica tridimensional. El análisis de las medidas del perfil hídrico, realizadas a diferentes distancias del tronco del árbol sugiere la necesidad de utilizar un método de ponderación. El método propuesto en el presente trabajo no es más que una aproximación, ya que un estudio más detallado de transferencia no es compatible con una experimentación a gran escala.

Finalmente, una dificultad esencial hallada en este trabajo proviene de las propias características de la planta en estudio. Las escasas diferencias de ETR encontradas durante los cuatro años de experimentación mencionados, entre los dos marcos de plantación, pueden ser debidos al hecho de que el olivo no haya reaccionado de forma definitiva a las condiciones de riego. Está claro, por otra parte, que el fenómeno de la alternancia se ha mantenido, lo que está a favor de lo expresado por ciertos autores, de que el riego no hace desaparecer dicho fenómeno, al menos a corto plazo. Por último, diremos que esta alternancia y los azares inevitables climáticos hacen muy arriesgado el establecer conclusiones rigurosas sobre la producción, lo que exigirá un estudio más prolongado.

Recu le 10 septembre 1987. Accepté le 6 avril 1988

\section{AGRADECIMIENTO}

Los autores agradecen a los Ingenieors Técnicos J. LIÑÃN y J. Prieto el control de la producción. El presente trabajo forma parte de un proyecto subvencionado por la CAICYT (España) y se ha realizado en el marco de una Acción Integrada Hispano-Francesa entre el IRNAS (C.S.I.C.) y el IMG (C.N.R.S.). 


\section{BIBLIOGRAFIA}

Bloemen G. W., 1980. Calculation of hydraulic conductivities of soils from texture and organic matter content. Z. Pflanzenernähr. Bodenk., 143, 581-605.

Bourdelles J. Le, 1979. Maîtrise des apports d'eau par aspersion et par goutte à goutte. Résultats d'essais sur agrumes, oliviers et pêchers. Proc. Seminaires sur l'irrigation localisée, II, 293-310. Bologne, Italie.

Bourdelles, J. Le, 1986. L'utilisation de l'eau en oléiculture. Résultats obtenus. Orientations nouvelles de la recherche. Olea, 17 (décembre), 189-193.

Cruz-Condé J., Fuentes M., 1986. Riego por goteo del olivar : dosis de agua. Olea, 17 (diciembre), 203-205.

Fernandez J. E., Moreno F., Cabrera F., Martin Aranda J., Arambarri P., 1987. Experiencias con $\mathrm{P}^{32}$ para el seguimiento de la extracción de agua por el olivo regado por goteo. Aceptado en el VII Congreso Nacional de Qúimica (Quimica Agrícola y Alimentaria). Sevilla, octubre 1987.

Genuchten M. Th. Van, 1980. A closed-form equation for predicting the hydraulic conductivity of unsaturated soils. Soil Sci. Am. J., 44, 892-898.

Haverkamp R., Parlange J. Y., 1986. Predicting the water retention curves from particle-size distributions. 1. Sandy soils without organic matter. Soil Sci., 142 (6), 325-339.

Hillel D., Krentos V. D., Stilianou Y., 1972. Procedure and test of an internal drainage method for measuring soil hydraulic characteristics in situ. Soil Sci., 114, 395-400.

Laty R., Vachaud G., 1987. AIDHYS 1, logiciel de traitement de mesures hydriques du sol. Institut de Mécanique de Grenoble. Grenoble, France. $33 \mathrm{p}$.

Martin Aranda J., Nuñez I., Moreno F., Arrue J. L., Roca M., 1982. Localized fertigation of no-tillage intensive young olive fields. Proc. 9th Conf. Intern. Soil Tillage Research Organization (ISTRO), I, 357-362.Osÿek; Yugoslavia.
Moreno F., Vachaud G., Martin Aranda J., 1983. Caracterización hidrodinámica de un suelo de olivar. Fundamento teórico y métodos experimentales. Anal. Edaf. Agrobiol., 42, 695-721.

Moreno F., Martin Aranda J., Rodriguez J., Vachaud G., Vauclin M., Laty R., 1987. Balance hidrico de un olivar con riego gota a gota. Memoria final de una Acción Integrada Hispano-Francesa. IRNAS (C.S.I.C.), Sevilla, España. 105 p.

Nuñez I., Arrue J. L., Moreno F., Martin Aranda J., 1981. Sustracción de humedad en la zona radicular del olivo (variedad manzanilio). Técnicas de seguimiento y primeros resultados obtenidos. Avances sobre la Investigación en Bioclimatologia, I, 515-524. Ed. CSIC (Madrid, España).

Serrano J. M. F., Abela V., 1982. Premiers résultats de l'essai d'irrigation goutte à goutte sur la variété Galega vulgar à l'ex-Station d'Oliviculture d'Elvas. Séminaire International sur l'utilisation des eaux "chargées" en Oléiculture. Tunis, octobre 1982. INIAERDépartement d'Oliviculture d'Elvas. Portugal.

Serrano J. M. F., Abela V., 1983. Travaux préliminaires à une sélection clonale de cinq variétés d'olivier de la Station d'Oliviculture d'Elvas. INIAER-Département d'Oliviculture d'Elvas, Portugal.

Serrano J. M. F., Abela V., 1984. Comportement de la variété Galega vulgar à l'irrigation goutte à goutte. Bilan de 4 années d'observations. Olea, 3-10 (June, 1984).

Serrano J. M. F., Delteil D., 1981, Résultat de l'essai d'irrigation goutte à goutte sur la variété Blanqueta présentée à la réunion technique du réseau "Irrigation en Oléiculture ". Bastia, Corse (mai, 1981).

Vachaud G., Passerat de Silans A., Balabanis P., Vauclin M., $1985 b$. Temporal stability of spatially measured soil water probability density function. Soil Sci. Soc. Am. J., 49, 822-828.

Vachaud G., Vauclin M., Riou C., Chaabouni Z., 1985a. Evapotranspiration en zone semi-aride de 2 couverts végétaux (gazon, blé) obtenue par plusieurs méthodes. II. Méthodes neutroniques et tensiométriques. Agronomie, 5 (3). 267-274 